\title{
In der Krise vereint? Milieuspezifische Perspektiven auf die Flüchtlingskrise
}

\author{
Raphael Kösters*, Heinrich-Heine-Universität Düsseldorf, Kommunikations- und Medienwissenschaft \\ Peter Obert, Heinrich-Heine-Universität Düsseldorf, Politikwissenschaft \\ Matthias Begenat, Center for Advanced Internet Studies (CAIS) \\ Olaf Jandura, Heinrich-Heine-Universität Düsseldorf, Kommunikations- und Medienwissenschaft \\ *Corresponding author: raphael.koesters@phil.uni-duesseldorf.de
}

\begin{abstract}
Kommunikationswissenschaftliche Forschung zur Fragmentierung konzentriert sich auf die Identifikation gesellschaftsweit geteilter Themen. Im vorliegenden Beitrag wird mit der sogenannten Flüchtlingskrise bewusst ein Thema als empirischer Fall gewählt, das als Krise bevölkerungsübergreifend als relevant wahrgenommen wird. Zur Klärung der Frage, ob die Auseinandersetzung über ein gemeinsames Thema zur Integration beiträgt, werden gruppenspezifische Deutungen der Flüchtlingskrise in differenten politisch-kommunikativen Milieus untersucht. Auf der Basis einer im Mai 2016 durchgeführten repräsentativen Online-Befragung ( $n=1488$ ) wurden mittels einer Clusteranalyse zwölf Milieus identifiziert, die sich hinsichtlich ihrer politischen Wertorientierungen sowie ihrer Nähe bzw. Distanz zur Politik unterscheiden. Die Themendeutungen, die über offene Assoziationstests erhoben wurden, lassen sich anhand einer Hauptkomponentenanalyse zu milieuspezifischen Perspektiven verdichten. Befunde zu Milieus mit extremen Grundhaltungen sprechen - trotz des geteilten Themas - gegen eine integrative Wirkung der Auseinandersetzung. Für gemässigte Milieus, die zwar ebenfalls spezifische Deutungen aufweisen, in denen aber auch Perspektiven anderer Bevölkerungsgruppen präsent sind, lässt sich ein integratives Potenzial erkennen.
\end{abstract}

\section{Keywords}

Öffentlichkeit, Integration, Milieus, Flüchtlingskrise, Deutungsanalyse

\section{$1 \quad$ Einleitung}

In der Kommunikationswissenschaft wird aus der Perspektive funktionaler Wirkungstheorien sowie einem öffentlichkeitstheoretischen Blickwinkel nach den Gelingensbedingungen einer kommunikativen Integration in zunehmend heterogenen Gesellschaften gefragt. Gleiche Themenagenden verschiedener Bevölkerungsgruppen in einer segmentär und stratifikatorisch ausdifferenzierten Gesellschaft fungieren dabei als Indikator für eine funktionierende Synchronisation über gemeinsame Themen, die überwiegend durch journalistische Angebote bereitgestellt werden (Jarren, 2000; Vlašić, 2004). Ein gemeinsames Themenrepertoire gilt als eine Grundvoraussetzung für den Austausch zwischen den Bürgern und die Wahrneh- mung von unterschiedlichen Perspektiven auf die Themen sowie für die Verortung des einzelnen Bürgers im öffentlichen Raum (Imhof, 2013). Zu diskutieren ist hierbei, ob das gemeinsame Themenrepertoire als Indikatorfür den Austausch bereits ausreicht, oder ob das Integrationspotenzial erst über die tatsächliche Kenntnis verschiedener Perspektiven beurteilt werden kann.

Mit der zunehmenden Angebotsfragmentierung (Napoli, 2014) und vor allem durch die steigende Bedeutung der politischen Online-Kommunikation (Vowe, 2017) wird aufgrund individualisierter Mediennutzung eine zunehmende Heterogenisierung der Bevölkerungsagenden befürchtet. Eine solche Differenzierung wird als dysfunktional für das demokratische System erachtet, da die eben genannten Funktionen nicht mehr erfüllt werden 
können (Eilders, 2013; Gerhards, 1998). Dies gilt sowohl für Normalzeiten des politischen Betriebs, die als Routinephasen definiert werden, als auch gerade für sogenannte Spezialzeiten (Wolling, 2005), die vom Bürger eine erhöhte Aufmerksamkeit gegenüber dem politischen Prozess verlangen. Hierzu zählen einerseits Wahlkämpfe, da in ihnen die Verteilung politischer Macht auch kommunikativ ausgehandelt wird und andererseits politische und gesellschaftliche Krisen, in denen Handeln und Entscheidungen der politischen Elite auf Widerspruch in relevanten Teilen der Bevölkerung stösst oder an einem sozialen Brennglasthema die heterogen verteilten Positionen in der Bevölkerung mehr als sonst deutlich zum Vorschein und in Konflikt zueinander treten (Imhof, 2011; Zaller, 2003).

Die so genannte Flüchtlingskrise wird im öffentlichen Diskurs als ein solches Brennglasthema angesehen (z. B. Gabriel, 2016). Spätestens seitdem im September 2015 eine grosse Zahl Flüchtlinge über Ungarn und Österreich nach Deutschland einreiste, wurde dieses Thema wie kaum ein anderes quer durch Parteien, Massenmedien, Online-Angebote und Gesellschaftsbereiche kontrovers diskutiert und erreichte eine hohe gesellschaftsweite Aufmerksamkeit (z.B. infratest dimap, 2015). Die kontroversen Diskussionen führen zur Befürchtung, dass sich an diesem Thema das zuvor «zur Ruhe gekommene Land» (Petersen \& Mayer, 2017) politisch spalte, was u.a. an der Partei "Alternative für Deutschland» (AfD) als neue politische Kraft festgemacht wird, die das Thema Migration stark bedient (Häusler, 2016).

An dieser Stelle wird eine zu untersuchende Leerstelle zwischen den Annahmen gegenüber der schwindenden Integrationskraft der Medien und der sozialen Realität während der Flüchtlingskrise deutlich. Folgt man der Literatur, so wird allgemein eine schwindende Integrationskraft durch eine Zersplitterung des Medienpublikums und damit einhergehend durch eine Zersplitterung der Bevölkerungssagenden ausgelöst. Es gebe keine gemeinsamen Themen mehr, die eine Grundlage für soziale Kohäsion bil- den können. Weniger gemeinsam erlebte Erfahrungen mögen zu weniger Anknüpfungspunkten für Gespräche und weniger Kontakten mit Andersdenkenden führen. Es könnte sich aufgrund fehlender gesellschaftsweiter Aushandlungsprozesse in der Folge weniger Verständnis für andere Positionen und Problemdefinitionen entwickeln (Übergang einer grundsätzlichen Fragmentierung hin $\mathrm{zu}$ einer Polarisierung). Einzelne Bürger sowie soziale Gruppen entfernen und entfremden sich voneinander (im Überblick: Stark, 2013; van Aelst et al., 2017). Für das Thema Flüchtlingskrise ist jedoch empirisch keine Zersplitterung der Agenda verschiedener Bevölkerungsgruppen erkennbar (Kösters \& Jandura, 2018) und dennoch wird eine fehlende Integration bis hin $\mathrm{zu}$ einer politischen Spaltung des Landes debattiert (z. B. Arlt \& Wolling, 2017; Köcher, 2016; Vowe, 2016).

Diese Leerstelle ist zunächst aus einem methodisch-konzeptionellen Blickwinkel zu erklären, wenn man die Erhebung der Themenagenden der Bevölkerung genauer betrachtet. Diese wird zumeist über die offene "most important problem"-Frage (Stark, 2014) oder über eine Bedeutungseinschätzung relevanter Themen auf einer Rang- oder einer Likertskala erfasst (z.B. Haas \& Brosius, 2013). Implizit wird davon ausgegangen, dass sich hinter den Themennennungen oder Relevanzbewertungen ein zumindest ähnliches Themenverständnis bzw. eine ähnliche Themendeutung steht. Argumente gegen diese Annahme lassen sich aus zwei theoretischen Perspektiven heraus formulieren. Die erste Perspektive nimmt die massenmediale Berichterstattung als Ausgangspunkt: Hier geht es um Studien, die in die Forschungstradition des Second LevelAgenda-Settings (López-Escobar et al., 1998) oder des Attribute-Agenda-Settings (Besova \& Cooley, 2009) einzuordnen sind. In dieser Weiterentwicklung der AgendaSetting-These wird davon ausgegangen, dass die Medienberichterstattung nicht nur die Salienz und Aufmerksamkeit gegenüber Themen beeinflusst, sondern über die Attribute in der Berichterstattung zu einem Thema auch die Perspektiven der 
Wahrnehmung beeinflusst (McCombs \& Shaw, 1993). Als Attribute werden dabei alle Merkmale definiert, die ein Objekt, also eine Person oder ein Thema in der Berichterstattung charakterisieren (McCombs, 2004). Empirische Studien können auf der Makro-Ebene den Zusammenhang zwischen der medialen Attribuierung von Themen und den Einstellungen der Bevölkerung zu diesen Themen zeigen (Besova \& Cooley, 2009, S. 224f.). Dies leitet über zur zweiten Perspektive - die der Medienrezipienten: Der lebensweltliche Kontext von Bevölkerungsgruppen und ihre politischen Prädispositionen haben einen hohen Einfluss auf die Auswahl von Medienangeboten sowie auf die Rezeption und Verarbeitung ihrer (politikbezogenen) Inhalte (Knobloch-Westerwick, 2014; Begenat, 2016). Nimmt man diese beiden theoretischen Zugänge zusammen ist prinzipiell davon auszugehen, dass sich hinter scheinbar geteilten Themen ganz unterschiedliche Deutungen verbergen. Die vorliegende Studie möchte einen Beitrag zu dieser zweiten Perspektive leisten.

Der Gedanke der Erfassung der Attribute $\mathrm{zu}$ einem Thema lässt sich mit der Frage nach dem Stand der gesellschaftlichen Integration verbinden. Während die Fragmentierungsforschung sich bislang auf die Identifikation von gemeinsamen Themen konzentriert hat (u. a. Haas \& Brosius, 2013; Stark, 2014), möchte die vorliegende Studie einen Schritt weitergehen. Der Beitrag fragt explorativ danach, ob sich hinter dem bevölkerungsübergreifend gemeinsam als wichtig wahrgenommenen Thema je nach Bevölkerungsgruppe gleiche oder unterschiedliche Beurteilungskriterien wiederfinden, die einer gesamtgesellschaftlichen Integration zuträglich sind oder eben nicht. Zur Klärung dieser Frage greifen wir auf einen Ansatz auf der Meso-Ebene zurück und untersuchen die Homogenität bzw. Heterogenität der gruppenspezifischen Deutungen der Flüchtlingskrise in verschiedenen politisch-kommunikativen Milieus (Mahrt \& Begenat, 2013; Weiß, 2009, 2013). ${ }^{1}$

1 Die Autoren bedanken sich bei Yannik Maihoff sowie Benedikt Mensing für die Unterstützung bei der Auswertung.

\section{Theorie}

\subsection{Politische Heterogenität als Heraus- forderung einer integrierten Öffent- lichkeit}

Die Frage, was eine demokratische und pluralistische Gesellschaft zusammenhält, wird in verschiedenen Disziplinen für jeweils unterschiedliche Sphären, wie etwa die Rechtsordnung, diskutiert (für einen Überblick siehe Vlašić, 2004, S. 16 ff.). Die Kommunikationswissenschaft betrachtet die Integrationsfunktion, die die Öffentlichkeit für eine sozial und politisch heterogen ausgeformte Gesellschaft leisten kann. Mediale Angebote können dabei integrierend wirken, in dem sie erkennbar machen, welche Themen und Problemsichten «sozial virulent sind und welche Problemlösungen aus welchen Gründen bei wem auf hinreichende Akzeptanz stoßen - und welche nicht» (Neidhardt, 1994, S. 26). Vorausgesetzt wird dabei, dass relevante politische Angelegenheiten «im Horizont aller Bürger» (Gerhards, 1998, S. 40) erscheinen und dass die verschiedenen sozialen Gruppen, die am meinungs- und willensbildenden Streit um das Gemeinwohl teilnehmen, die Bereitschaft zeigen, auch Problemsichten und Lösungsvorschläge anderer Teilgruppen zu respektieren und in ihrer eigenen Meinungsbildung zu berücksichtigen (Imhof, 2011).

Die erste Voraussetzung betrifft dabei den Kern der Fragmentierungsdebatte, die zweite stellt zur Diskussion, inwiefern gruppenspezifische Medienrepertoires und eine gruppenspezifische Verarbeitung von Themen, neben der gesellschaftsweiten Wahrnehmung auch die wechselseitige Kenntnisnahme politischer Positionen anderer Bevölkerungsgruppen einschränken. Das öffentlichkeitstheoretische Idealbild verlangt in diesem Zusammenhang keinesfalls, eine gesellschaftsweite Meinungskonsonanz herzustellen. Vielmehr geht es darum, dass in gruppenspezifischen Teilöffentlichkeiten bzw. MedienArenen (Ferree et al., 2002) durch eine an Pluralität und Diskursivität orientierte Berichterstattung die verschiedenen Positionen zu politischen Themen prinzipiell anerkannt und für alle einsehbar abgebil- 
det werden. Diese Idee einer «Integration durch Konfliktkommunikation» kann letztlich die Orientierungsfunktion stärken, die wechselseitige Toleranz divergierender Problemsichten auf Seiten der Bevölkerung und insgesamt die Vernetzung heterogener Gesellschaften begünstigen (Vlašić, 2004; Wessler, 2002a, 2002b).

Dass die sogenannte «Flüchtlingskrise» als empirisch zu untersuchendes Thema gesellschaftsübergreifend wahrgenommen wird, verschiebt den Fokus des vorliegenden Beitrags auf die Untersuchung der gruppen- bzw. milieuspezifischen Verarbeitung. Durch die besondere Charakteristik des Krisenthemas, ist die Frage, ob die öffentliche Auseinandersetzung darüber im Horizont aller Bürger erscheint, bereits beantwortet. $\mathrm{Ob}$ die milieudifferente Verarbeitung und Deutung des gemeinsamen, gesellschaftsweit geteilten Themas allerdings integrierend wirkt, bleibt zu klären.

Dass sich unterschiedliche Teilgruppen der Bevölkerung Themen auf ihre eigene Art und Weise aneignen und diese unterlegt durch ihre eigenen politischen Prädispositionen - verarbeiten, ist in der in der kommunikationswissenschaftlichen Forschung unumstritten. Prominent ist hierbei der Verweis auf von Bennett und Iyengar (2008) als "stratamentation» bezeichnete Tendenzen. Diese umfassen zum einen vertikale Stratifikationsprozesse, nach denen sich Teile der Bevölkerung aus der politisch-kommunikativen Teilhabe gänzlich ausklinken, andere Bevölkerungsgruppen hingegen besonders stark involviert sind. Teilgruppen der Gesellschaft wenden sich demnach sehr häufig politischen Informationsangeboten $\mathrm{zu}$ und setzen sich vertieft mit deren Inhalten auseinander, andere Gruppen tun dies wiederum nur sporadisch und beiläufig oder gar überhaupt nicht. Ausgehend davon basiert die politische Meinungsbildung je nach Bevölkerungsgruppe auf ganz unterschiedlichen Voraussetzungen (Schneider \& Eisenegger, 2016). Insbesondere unter den Hochinvolvierten können zudem horizontale Segmentierungsprozesse entlang von politischen Grundorientierungen identifiziert werden, die in weltanschaulicher Hinsicht die Medienselektion, Rezeption und Verarbeitung von Sachverhalten beeinflussen (Begenat, 2016; Knobloch-Westerwick, 2014; Yeo et al., 2015).

Verbindet man diese beiden Tendenzen mit der Entwicklung hin zu einem immer ausdifferenzierteren und an spezifischen (politischen) Zielgruppen orientierten Medienangebot, so besteht die Gefahr der Ausbildung von inhaltlich homogenen, politischen Teilöffentlichkeiten (u. a. Hall Jamieson \& Cappella, 2008; Stroud, 2011). Diese stellen den Gegensatz einer integrierten politischen Öffentlichkeit dar, weil das Einfinden in diesen als «Echokammern» bezeichneten Teilöffentlichkeiten die politische Abschottung von gesellschaftlichen Gruppen begünstigen kann. Innerhalb dieser politisch-medialen «Enklaven» scheinen, so die Argumentation, die Themen und Problemhaltungen anderer Bevölkerungsgruppen kaum wahrgenommen zu werden und falls doch, dann in abwertender Art und Weise. Ferner wird angenommen, dass kaum direkter kommunikativer Austausch über die Grenzen dieser Teilöffentlichkeiten hinaus stattfindet. Darin liegt eine Gefahr für das integrierende Element von Öffentlichkeit, weil mit einer permanenten Bestätigung bestehender Meinungen die Polarisierung von Problemhaltungen sowie eine abnehmende Bereitschaft zum offenen Diskurs verbunden sein kann (Prior, 2013; Sunstein, 2007). Der vorliegende Beitrag macht es sich nun aber nicht - wie zahlreiche Fragmentierungsstudien - zur Aufgabe, auf der Ebene der Medienrepertoires oder der Themen nach Überschneidungen und Gemeinsamkeiten zu suchen. Indem wir durch die betrachtete Krise ein Thema für die empirische Analyse wählen, das alle Bevölkerungsgruppen als virulent wahrnehmen und das - in der Umkehrung der Fragmentierungsthese - integrativ wirken könnte, ist es für uns möglich, die Analyse auf die Ebene der Themendeutungen zu erweitern und damit zu schärfen. Hierfür ist es zunächst notwendig, verschiedene Bevölkerungsgruppen zu identifizieren. 


\subsection{Der Milieu-Ansatz zur empirischen Erfassung von Tendenzen der "stratamentation"}

Wie kann die politische Heterogenität moderner Gesellschaften beschrieben werden bzw. in welchen Gruppen sollte überhaupt nach der Wahrnehmung von Themen und deren Deutungen gesucht werden? Wir orientieren uns zur Beschreibung der gesellschaftlichen Heterogenität am Konzept politisch-kommunikativer Milieus nach Weiß (2009, 2013). Dieses baut auf Erkenntnissen aus dem Bereich der (politischen) Soziologie (u.a. Hradil, 2006, Neugebauer, 2007) auf und unterstreicht die Nützlichkeit eines milieutheoretischen Zugangs für Fragestellungen der politischen Kommunikationsforschung. Politisch-kommunikative Milieus sind definiert als gesellschaftliche Teilgruppen, in denen politische Wertorientierungen und Einstellungen, Sozialisationserfahrungen, Partizipationsmuster, aber auch Informationsrepertoires sowie Modi politischer Kommunikation (z.B. unterschiedliche Routinen der Informationsverarbeitung sowie Differenzen in der Auseinandersetzung mit politischen Inhalten in der interpersonalen Kommunikation, siehe oben) systematisch miteinander verbunden sind. Die einzelnen Faktoren des Eigenschaftsprofils von Milieus (sozialer Ort, politische Grundorientierungen, Kommunikations- und Partizipationspraktiken) bilden insgesamt ein aufeinander abgestimmtes Gesamtbild. Milieus bezeichnen Gruppen Gleichgesinnter, "die die gesellschaftlichen Verhältnisse und die politische Welt ähnlich interpretieren und gestalten wollen - und sich darin von den Angehörigen anderer Milieus unterscheiden» (Weiß, 2013, S. 208). Die Einbindung des lebensweltlichen Kontextes und von handlungsleitenden Wertorientierungen in milieutheoretischen Analysen gewährt einen vertieften Einblick in subjektive Prinzipien der Verarbeitung von (medialen) Informationen zu politischen Sachverhalten. Es ist davon auszugehen, dass einerseits die milieuspezifischen Grundhaltungen gegenüber der Politik sowie andererseits weltanschaulich moderierte Formen der Mediennutzung und die
Rezeption entsprechender Inhalte die gruppenspezifischen Deutungen von politischen Themen in besonderem Masse prägen (Weiß, 2000).

Entlang der Grundorientierungen der Milieus offenbaren sich erhebliche Unterschiede, welche Anliegen Personen dauerhaft umtreiben und beschäftigen. Eben solche bedeutsamen Problemdefinitionen und die damit verwobenen milieuspezifischen Perspektiven werden dabei zu vorrangigen Beurteilungskriterien. Neben die Differenzierung der Themenrelevanzen tritt die Frage, was die Relevanz eines Themas ausmacht (Weiß 2013, S. 209; Weiß \& Jandura, 2017, S. 18). Milieus, die eine starke wirtschaftsliberale Grundhaltung einnehmen, machen etwa Kosten-Nutzen-Abwägungen und das Leistungsprinzip zum Massstab ihrer Bewertungen während andere Milieus Themen primär vor dem Hintergrund der Frage bewerten, was die sozial gerechteste Lösung sei (Begenat, 2017, S. 199). Ferner sind Unterschiede hinsichtlich der Offenheit gegenüber konträren Haltungen und damit Grenzen eines vernetzenden Austausches durch milieuübergreifende Kommunikation trotz teilweise übereinstimmender Themenhorizonte zu erwarten (Begenat, 2016, 2017).

Das medienseitige Pendant $\mathrm{zu}$ den beschriebenen individuellen oder gruppenspezifischen Grundhaltungen wird von der Forschung als Value-Frame bezeichnet. Solche wertebezogenen Deutungsmuster liegen in der Berichterstattung vor, «wenn Werte, die in der politischen Kultur sedimentiert sind, als übergeordnete Bezugsrahmen für Sachverhalte, Vorgänge oder Akteure fungieren» (Scheufele et al. 2012, 432; siehe u.a. auch Eilders 2004; Scheufele \& Engelmann 2014). Laut Schemer et al. (2012, S. 338; siehe auch Ball-Rokeach \& Loges 1996; Lee et al. 2008) ist es charakteristisch für die journalistische Arbeit, politische Auseinandersetzungen entlang von in Konflikt stehenden, wertebezogenen Grundorientierungen zu organisieren. Es ist vor diesem Hintergrund davon auszugehen, dass wertebezogene Grundorientierungen der politischen Informationsnutzung nicht nur als Einflussfaktor (etwa bei der Medienselektion und 
Verarbeitung medialer Inhalte) vorausgehen, sondern dass Medieninhalte ebenso einen Anteil an der Sozialisation und Aktualisierung von politischen Grundhaltungen haben (Rippl, 2008).

Zur Charakterisierung von Milieus und deren Lebenswelten wählt man üblicherweise im ersten Schritt eine typologisierende Herangehensweise, um im zweiten Schritt die Eigenschaften der identifizierten Typen in den sonstigen relevanten Beschreibungsdimensionen (u.a. Kommunikationspraxis, soziale Verortung, Themenagenden) näher $\mathrm{zu}$ betrachten. Der Fokus im Rahmen der Typologisierung liegt auf zwei Elementen, (a) handlungsleitenden Weltanschauungen sowie (b) der grundsätzlichen Zuwendung oder Abkehr von der politischen Sphäre, das heisst auf den Dimensionen der Segmentierung und der Stratifikation, die der Begriff der «stratamentation» zusammenfassend kennzeichnet (Mahrt \& Begenat, 2013). Weltanschauungen können über die individuelle bzw. gruppenspezifische Positionierung hinsichtlich relevanter Wertekonflikte einer politischen Kultur bestimmt werden. Die hier betrachtete deutsche Gesellschaft ist primär durch zwei Konfliktlinien und davon abgeleitete Orientierungen geprägt: Auf der ersten Konfliktlinie steht eine marktliberale bzw. auf den individuellen Nutzen ausgerichtete Orientierung einer sozialstaatlichen-egalitären Orientierung gegenüber (sozioökonomische Konfliktlinie); auf der zweiten Konfliktlinie bildet eine gesellschaftspolitisch libertäre und partizipative Orientierung den Gegenpol einer autoritären, auf die Durchsetzung von Recht und Ordnung ausgerichteten Orientierung (politisch-kulturelle Konfliktlinie) (für eine Übersicht zu relevanten Konfliktlinien siehe etwa Niedermayer, 2009). Das zweite für ein Milieu konstitutive Element kann durch die mehr oder weniger grosse Nähe oder Distanz zur Politik auf Seiten von einzelnen Individuen bzw. Gruppen beschrieben werden. Beide typenbildenden Elemente bestimmen zusammengenommen die grundsätzliche, milieuspezifische Haltung gegenüber dem politischen System und politischen Sachverhalten.
Vor dem dargestellten theoretischen Hintergrund geht der vorliegende Beitrag explorativ vor und beantwortet die folgenden drei Forschungsfragen (FF):

, FF1: Welche Deutungsmuster zur «Flüchtlingskrise» lassen sich auf Seiten der Bevölkerung identifizieren?

, FF2: Welche Deutungsmuster zur «Flüchtlingskrise» sind in welchen Milieus dominant?

, FF3: Wie können Deutungsmuster vor dem Hintergrund der milieuspezifischen Lebenswelt interpretiert werden?

\section{Methodisches Vorgehen}

Das methodische Vorgehen unserer Untersuchung umfasst verschiedene Komponenten, die schematisch in Abbildung 1 dargestellt sind. Zur Untersuchung der Kommunikationspraktiken politischer Milieus in Deutschland, wurde zunächst mithilfe des Access-Panels eines Marktforschungsunternehmens im Mai 2016 (Feldzeit: 23. bis 30.05.2016) eine Online-Befragung $(n=1488$, quotierte und bereinigte Stichprobe nach Alter, Geschlecht, Bildung, Region, Berufstätigkeit, gewichtet nach Bildung und Alter) unter der wahlberechtigten Bevölkerung zwischen 18 und 69 Jahren durchgeführt. Der Repräsentationsschluss der Studie gilt demnach nicht für die Gesamtbevölkerung. Ein Abdeckungsfehler ergibt sich für Nicht-Onliner sowie Personen über 70 Jahre. Gegenstand der Erhebung waren dabei sowohl politische Grundhaltungen und die Eigenpositionierung auf der politisch-kulturellen und sozioökonomischen Dimension, als auch eine umfassende Erfassung des Mediengebrauchs und des interpersonalen Kommunikationsverhaltens der Befragten. Die Ausgestaltung des Fragebogens orientiert sich dabei an etablierten Ansätzen aus dem Bereich der politischen Soziologie und Kommunikationsforschung. Bei der Typologisierung politisch-kommunikativer Milieus wird zwischen zwei Dimensionen unterschieden: der vertikal-stratifikatorischen Ebene auf der einen und der horizontal-segmen- 
Abbildung 1: $\quad$ Übersicht zu den methodischen Komponenten der Studie

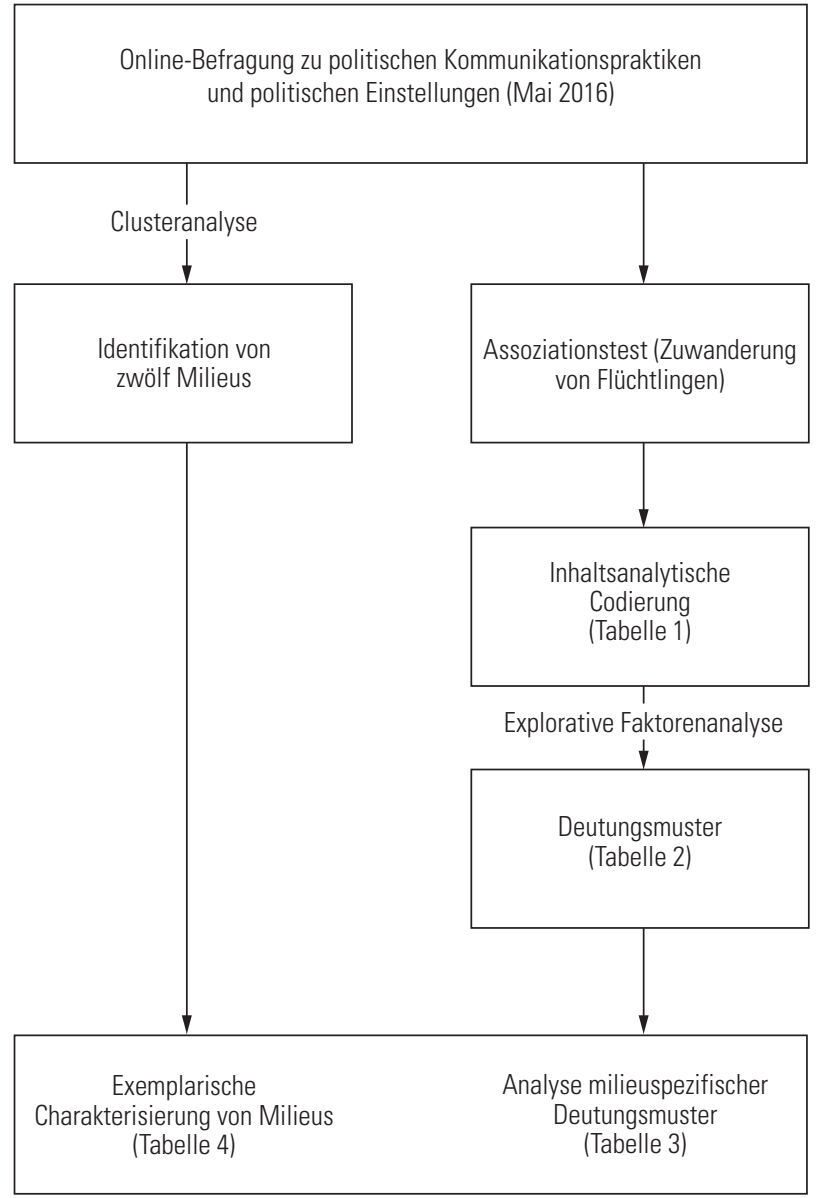

tierenden Ebene auf der anderen Seite. Bevor diese beiden Analyseebenen herangezogen werden, um Ähnlichkeitsgruppen mittels einer Clusteranalyse $\mathrm{zu}$ bestimmen, die weiterführend als politisch-kommunikative Milieus interpretiert werden, sollen zunächst konzeptuelle Kategorien und empirische Variablen zur Analyse der entsprechenden Gruppenunterschiede beschrieben werden.

Auf der vertikal-stratifikatorischen Ebene wird zur Beschreibung der staatsbürgerlichen Rolle die konzeptuelle Dimension der Nähe/Distanz zur politischen Sphäre herangezogen. Im Vordergrund steht dabei vor allem die Erfassung der individuellen politischen Kompetenz- und der Einflussüberzeugungen (political efficacy). Hierbei geht es um die Einschätzung der Bürger hinsichtlich ihrer Fähigkeit «politische Vorgänge verstehen und durch individuelles politisches Engagement beeinflussen zu können» (Vetter, 1997, S. 53). Für die Differenzierung der Milieus auf der stratifikatorischen Dimension werden sowohl die individuelle Kompetenzeinschätzung, dass der eigenen Person Fähigkeiten zur Verfügung stehen, politische Sachverhalte $\mathrm{zu}$ verstehen und sich wirksam beteiligen zu können (internal efficacy), als auch das Responsivitätsvertrauen gegenüber politischen Eliten erhoben (external efficacy). Die Messung beider Aspekte des Konstrukts der wahrgenommenen politi- 
schen Selbstwirksamkeit ermöglicht die Political Efficacy Kurzskala (PEKS) (Beierlein, Kemper, Kovaleva \& Rammstedt, 2014) mit jeweils zwei Items. ${ }^{2}$ In den erhobenen Daten lässt sich für die Konsistenz der internen Dimension ein Cronbachs Alpha-Wert von 0,78 und für die externe Dimension von 0,76 feststellen. Im Vergleich zur häufigen Operationalisierung über das politische Interesse (z. B. Mahrt \& Begenat, 2013; Weiß, 2013) erachten wir das zweidimensionale Konzept der politischen Selbstwirksamkeit für die stratifikatorische Differenzierung als besser geeignet, weil es bedeutsame Unterschiede zwischen gesellschaftlichen Gruppen erkennbar macht. Durch die alleinige Erfassung einer allgemeinen Kategorie wie dem politischen Interesse könnten nämlich für einzelne Gruppen charakteristische Kombinationen, z.B. von einem hohen politischen Engagement und einer gleichzeitig hoch ausgeprägten Distanz gegenüber der etablierten Politik, nicht identifiziert werden. Des Weiteren ist die Frage nach dem politischen Interesse aus methodischer Perspektive anfälliger für ein sozial erwünschtes Antwortverhalten.

Die horizontal-segmentierende Ebene erfasst im Rahmen der Differenzierung politisch-kommunikativer Milieus solche Einstellungen der Befragten, die sich jeweils als Wertepositionierungen auf der sozioökonomischen und politisch-kulturellen Dimension konzeptualisieren lassen. Im Vordergrund steht dabei insbesondere die Erfassung der individuellen Präferenzen in Form von Zustimmung oder Ablehnung bestimmter wertgeladener Positionen. Die abgefragten Items wurden auf Basis vorliegender Literatur und Befragungen zur Erfassung von politischen Wertorientierungen ausgewählt

2 Items der Skala der Internal Political Efficacy der PEKS waren: «Wichtige politische Fragen kann ich gut verstehen und einschätzen» und «Ich traue mir zu, mich an einem Gespräch über politische Fragen aktiv zu beteiligen.» Items der External Political Efficacy waren: «Die Politiker kümmern sich darum, was einfache Leute denken» und «Die Politiker bemühen sich um einen engen Kontakt zur Bevölkerung». (z. B. Neugebauer, 2007; World Values Survey, 2013) und im Rahmen einer Vorstudie $(\mathrm{n}=165$, Studierendensample) auf Validität und Reliabilität hin überprüft. Beide Konfliktdimensionen werden mit jeweils drei Items erhoben und Befragte wurden auf einer 6er-Skala gebeten, ihre Zustimmung oder Ablehnung anzugeben. ${ }^{3}$ Die theoretisch und konzeptuell formulierte Erwartung bezüglich der Zuordnung der dargestellten Items zu den zwei Konfliktdimensionen wurde mithilfe einer Hauptkomponentenanalyse $(\mathrm{KMO}=0,57$, $44 \%$ erklärte Varianz, Varimax-Rotation) überprüft. Dabei zeigt sich, dass die sechs Items tatsächlich sinnvollerweise auf zwei zugrundeliegende Faktoren reduziert werden können, die mit den beiden beschriebenen Dimensionen der Wertekonflikte korrespondieren. Für die weiterführende Analyse werden diese Items zu zwei Indizes zusammengefasst, die auszählen, bei wie vielen der drei Items für jede Konfliktdimension welche richtungspolitische Position eingenommen wurde.

3 Items zur sozioökonomischen Dimension umfassen: «Der Staat sollte mehr Verantwortung dafür übernehmen, dass jeder Bürger abgesichert ist.» vs. "Jeder einzelne sollte mehr Verantwortung für sich selbst übernehmen.»; «Zur Sicherung sozialstaatlicher Leistungen sollte die Politik Steuern und Abgaben erhöhen.» vs. «Die Politik sollte Steuern und Abgaben senken, auch wenn das weniger sozialstaatliche Leistungen bedeutet.»; «Der Staat sollte der Wirtschaft Vorgaben machen und auf deren Einhaltung drängen.» vs. «Die Wirtschaft soll sich ohne staatliche Eingriffe möglichst drei entfalten können.». Items zur politisch-kulturellen Dimension sind: «Die Freiheit der Bürger muss vor Eingriffen des Staates geschützt werden, selbst wenn dadurch der Schutz vor Kriminalität zurückstehen muss.» vs. «Um die Bürger vor Kriminalität zu schützen, hat der Staat das Recht, die Freiheit der Bürger einzuschränken.»; «Es ist wichtig, eine offene und tolerante Gesellschaft zu stärken.» vs. «Es ist wichtig, unsere eigene deutsche Kultur und Tradition zu pflegen.»; «Demokratische Beteiligung der Bürger ist immer wichtiger als eine starke politische Führung.» vs. «Um unsere Probleme im Land zu lösen, ist eine starke politische Führung wichtiger als demokratische Beteiligung der Bürger.». 
Die beschriebenen Variablen der segmentierenden und stratifikatorischen Ebene wurden in einem dritten Schritt einer hierarchischen Clusteranalyse unterzogen. Die Distanz zwischen den Clustern wurde auf Basis der quadrierten euklidischen Distanz berechnet und die Cluster mit Hilfe des Ward-Verfahrens aggregiert. Als Entscheidungskriterien über die Anzahl der Cluster dienten vor allem der sprunghafte Anstieg der Clusterheterogenität (Dendrogramm) sowie die Koeffizienten der F-Werte der jeweiligen Cluster die kleiner als 1 waren. Die Befunde wurden zusätzlich durch eine multiple Diskriminanzanalyse validiert. Diese hat gezeigt, dass eine Zwölf-Cluster-Lösung (siehe Abbildung 2) auf Basis statistischer Kriterien die existierende «stratamentation» der deutschen Bevölkerung am besten repräsentiert.

Darüber hinaus wurde in die standardisierte Befragung eine offene Frage in Form eines Assoziationstests integriert. Dabei wurden die Befragten in Anlehnung an die in der Sozialpsychologie angewandte "thought-listing technique» (Cacioppo et al., 1981) gebeten, die Frage zu beantworten: «Können Sie bitte in das offene Feld eingeben, woran Sie spontan denken, wenn über die Zuwanderung von Flüchtlingen nach Deutschland diskutiert wird?» Von dieser Frageformulierung erhofften wir uns einen Blick auf die Bandbreite der Assoziationen, die die Befragten mit dem Thema der Flüchtlingskrise verbinden. Über die Homogenität bzw. Heterogenität dieser Angaben lassen sich sowohl milieuspezifische Sichtweisen als auch Aussagen zur Offenheit gegenüber Deutungen anderer und damit zum integrierenden Potenzial des Themas ableiten. Für die Codierung der Antworten der Befragten wurde auf Basis themenbezogener Vorarbeiten zum Migrationsdiskurs (u. a. Helbling, 2014; Roggeband \& Vliegenthart, 2007) sowie Probecodierungen eigens ein Kategoriensystem entwickelt. Dieses differenziert zwischen einfachen, schlagwortartigen Antworten der Befragten auf der einen Seite und komplexeren, seitens der Befragten ausformulierten Deutungsmustern auf der anderen. Um auf Basis dieser Frage
Tabelle 1: Übersicht der Kategorien im Codierschema

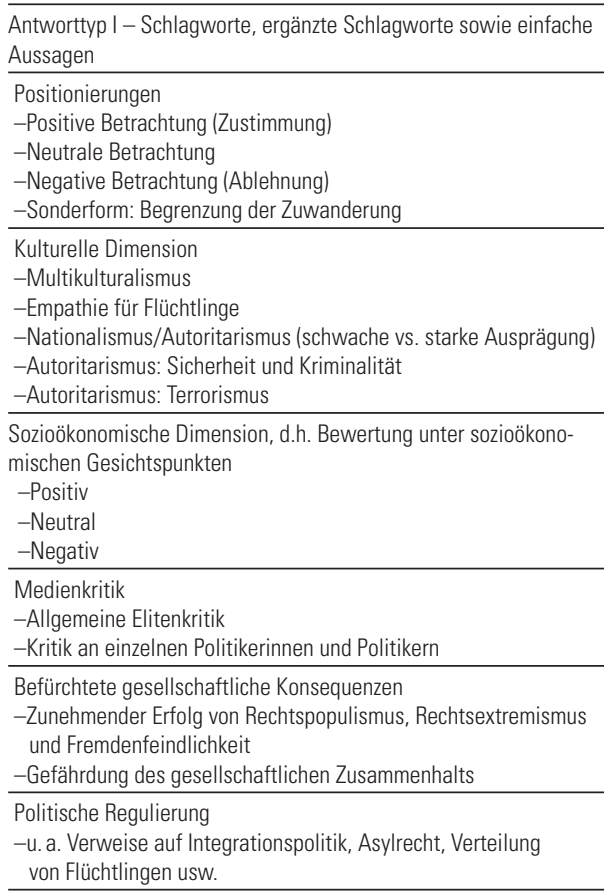

spezifische Deutungsmuster zu ermitteln, werden im Folgenden nur die Aussagen der Befragten, die dem ersten Antworttyp zugeordnet werden können (einfache Aussagen und Schlagworte), näher betrachtet. Insbesondere aus empirischer Sicht lässt sich feststellen, dass etwa $80 \%$ der Aussagen auf diesen Antworttyp entfallen. ${ }^{4}$ Im

4 Der zweite Antworttyp wird aufgrund der für eine differenzierte Auswertung nach Milieus zu geringen Fallzahl von $n=224$ nicht weiter betrachtet. Zur Codierung dieses Antworttyps waren die folgenden Ausprägungen möglich, die jeweils Deutungen umfassen, die für bestimmte Wertekonfliktpositionen stehen: Multikulturalismus und Humanität (libertärer Wertepol), Assimilation/Anpassung, Kulturelle Differenzen, Sicherheit/ Kriminalität/Terrorismus (jeweils autoritärer Wertepol), Zuwanderung von Flüchtlingen als ökonomische Chance (marktliberale Konfliktpol), Belastung des Sozialstaats und Sozialchauvinismus (sozialstaatlich-autoritäre Wertesynthese), Differenzierung von nützlichen und weniger nützlichen Zuwanderern (marktliberal-autoritäre Wertesyn- 
Rahmen der Codierung wurden die themenbezogenen Assoziationen der Befragten dichotom auf das Vorkommen bzw. Nicht-Vorkommen inhaltlich-thematischer Schwerpunkte bewertet (siehe auch Tabelle 1). Sowohl die Intra-Coder-Reliabilität (Krippendorffs Alpha: 0,8), als auch die Forscher-Codierer-Reliabilität (Krippendorffs Alpha: 0,7) liefern dabei mit Blick auf die hohe Komplexität der Codierung einen zufriedenstellenden Wert.

Das zentrale Ziel der Analyse der Antworten der Befragten aus dem Assoziationstest ist es Aussagen darüber zu generieren, inwiefern sich die häufig grundverschiedenen Antworten mithilfe von komplexitätsreduzierenden Verfahren $\mathrm{zu}$ themenbezogenen Deutungen verdichten lassen. $\mathrm{Zu}$ diesem Zweck wird eine Hauptkomponentenanalyse auf Basis einer tetrachorischen Korrelationsmatrix der zuvor dargestellten nominalskalierten dichotomen Variablen des ersten Antworttyps durchgeführt (siehe Tabelle 1). Die Ergebnisse der Analyse zeigen, dass sich insgesamt sieben Faktoren bzw. Deutungsmuster identifizieren lassen, wobei diese sich voneinander im Hinblick auf deren empirische Relevanz bisweilen sehr stark unterscheiden. ${ }^{5}$ Aus diesem Grund nehmen wir auf Basis des Varianzaufklärungs-

these) sowie libertär-solidarische bzw. autoritäre Elitenkritik. Eine Antwort dieses Typs, die beispielsweise mit der Ausprägung «Multikulturalismus \& Humanität» verschlüsselt wurde, lautet: «Ich bin für die Zuwanderung von Flüchtlingen, da wir unglaublich viel von ihnen lernen können. Wir stärken dadurch unsere Gemeinschaft und entdecken neue Möglichkeiten. Dafür muss den Flüchtlingen aber auch eine Chance gegeben werden.».

5 Die im Rahmen der Analyse ermittelten Faktorladungen der jeweiligen Items können dabei in Abhängigkeit von ihrem Wertebereich und Vorzeichen als zwei Pole einer inhaltlichen Dimension (hier als Deutungsmuster bezeichnet) interpretiert werden. Für die substanzielle Interpretation der Deutungsmuster bedeutet dies, dass Äusserungen, die dem positiven Pol zugeordnet werden können, häufiger vorkommen und Äusserungen, die dem negativen Pol zugeordnet werden können, tendenziell weniger häufig vorkommen (siehe Tabelle 6 im Anhang). potenzials der identifizierten Faktoren eine Differenzierung zwischen primären und sekundären Deutungsmustern vor. Als primäre Deutungsmuster bzw. Hauptperspektiven können die empathisch-offene Perspektive und die nationalistisch-ausgrenzende Perspektive gelten. Diese umfassen zusammengenommen knapp mehr als $50 \%$ der im Assoziationstest getätigten Aussagen. Sowohl die Häufigkeit des Vorkommens (hier mit Ausnahme der medialen Repräsentations-Perspektive, die hinsichtlich der Häufigkeit noch knapp vor der nationalistisch-ausgrenzenden Perspektive rangiert) als auch das Varianzaufklärungspotenzial der sekundären Deutungsmuster (im Wertebereich zwischen 5,7 und $10,9 \%$ ) sind deutlich geringer. Es lässt sich deshalb schlussfolgern, dass es sich hierbei - im direkten Vergleich mit den primären Deutungsmustern - um selten getätigte Aussagen handelt. Diese umfassen Perspektivierungen der Elitenkritik, der wahrgenommenen Chancen und negativen Folgen der Zuwanderung, Kritik der medialen Berichterstattung sowie eine (gemässigte) rechtspopulistische Perspektive (siehe Tabelle 2 sowie Tabelle 3 ).

\section{Ergebnisse}

\subsection{Typologie politisch-kommunikativer Milieus}

In unserer Untersuchung werden die unterschiedlichen Deutungen der Bevölkerung auf die Zuwanderung von Flüchtlingen entlang von politisch-kommunikativen Milieus differenziert. Ausgangspunkt dafür ist eine empirisch fundierte Milieu-Typologie, die im Folgenden kurz erläutert wird. Als Ergebnis der Clusteranalyse konnten wir zwölf Milieus identifizieren, die sich auf Grundlage ihrer Positionierung bei gesellschaftlich relevanten Wertekonflikten sowie ihrer Nähe bzw. Distanz zur Politik in einem zweidimensionalen Raster der «stratamentation» verorten lassen (siehe Abbildung 2). Abhängig von der pro Milieu jeweils spezifischen Kombination aus interner und externer politischer Selbstwirksamkeit können die einzelnen identifizierten $\mathrm{Ge}$ - 
Tabelle 2: Übersicht der Hauptkomponenten und entsprechende inhaltliche Variablen

\begin{tabular}{|c|c|c|c|c|}
\hline \multicolumn{2}{|c|}{$\begin{array}{l}\text { Faktor/Deutungs- } \\
\text { muster }\end{array}$} & \multirow{2}{*}{$\begin{array}{l}\text { Variablen - positiver Pol } \\
\text { Positive Betrachtung des Themas } \\
\text { Multikulturalismus } \\
\text { Empathie für Flüchtlinge } \\
\text { Erstarken Rechtspopulismus und } \\
\text { Fremdenfeindlichkeit }\end{array}$} & \multirow{2}{*}{$\begin{array}{l}\text { Variablen - negativer Pol } \\
\text { Negative Betrachtung des Themas } \\
\text { Negative Betrachtung aus sozioökon- } \\
\text { omischer Perspektive }\end{array}$} & \multirow{2}{*}{$\begin{array}{c}\text { Anteil erklärter Varianz } \\
21.11 \%\end{array}$} \\
\hline 1 & $\begin{array}{l}\text { Empathisch- } \\
\text { offene Perspek- } \\
\text { tive }\end{array}$ & & & \\
\hline 2 & $\begin{array}{l}\text { Nationalistisch- } \\
\text { ausgrenzende } \\
\text { Perspektive }\end{array}$ & $\begin{array}{l}\text { Begrenzung der Zuwanderung } \\
\text { Negative Betrachtung des Themas } \\
\text { Nationalismus/Autoritarismus (starke } \\
\text { Ausprägung) } \\
\text { Autoritarismus: Terrorismus } \\
\end{array}$ & Neutrale Betrachtung des Themas & $14.58 \%$ \\
\hline 3 & Elitenkritik & $\begin{array}{l}\text { Elitenkritik mit Bezug auf Konfliktdi- } \\
\text { mensionen }\end{array}$ & $\begin{array}{l}\text { Elitenkritik ohne Bezug auf Konflikt- } \\
\text { dimensionen }\end{array}$ & $10.90 \%$ \\
\hline 4 & $\begin{array}{l}\text { Negative Folgen- } \\
\text { Perspektive }\end{array}$ & $\begin{array}{l}\text { Autoritarismus: Terrorismus } \\
\text { Erstarken Rechtspopulismus und } \\
\text { Fremdenfeindlichkeit }\end{array}$ & Kritik an einzelnen Politikern & $10.31 \%$ \\
\hline 5 & $\begin{array}{l}\text { Chancen-Pers- } \\
\text { pektive }\end{array}$ & $\begin{array}{l}\text { Positive Betrachtung aus sozioökono- } \\
\text { mischer Perspektive }\end{array}$ & $\begin{array}{l}\text { Autoritarismus (starke Ausprägung) } \\
\text { Herausforderung für gesellschaftli- } \\
\text { chen Zusammenhalt }\end{array}$ & $9.24 \%$ \\
\hline 6 & $\begin{array}{l}\text { Mediale } \\
\text { Repräsentations- } \\
\text { Perspektive }\end{array}$ & Medienkritik & $\begin{array}{l}\text { Neutrale bis tendenziell negative } \\
\text { Betrachtung aus sozioökonomischer } \\
\text { Perspektive }\end{array}$ & $7.41 \%$ \\
\hline 7 & $\begin{array}{l}\text { Gemässigt } \\
\text { rechts- } \\
\text { populistische } \\
\text { Perspektive }\end{array}$ & $\begin{array}{l}\text { Nationalismus/Autoritarismus } \\
\text { (schwache Ausprägung) } \\
\text { Autoritarismus: Sicherheit und } \\
\text { Kriminalität } \\
\text { Neutrale bis tendenziell negative } \\
\text { Betrachtung aus sozioökonomischer } \\
\text { Perspektive } \\
\text { Regulierung }\end{array}$ & Begrenzung der Zuwanderung & $5.71 \%$ \\
\hline
\end{tabular}

sellschaftssegmente auf der vertikal-stratifikatorischen Dimension zu fünf Gruppen von Staatsbürgerrollen zusammengefügt werden. So kennzeichnet sich die Gruppe der Aktiven Staatsbürger beispielsweise durch ein hohes bis sehr hohes Vertrauen in die eigene politische Selbstwirksamkeit und erachtet die politischen Eliten als sehr responsiv. Demgegenüber stehen am unteren Ende dieser Dimension zwei Gruppen mit einer relativ grossen Distanz zur Politik (Entfremdete Demokratiekritiker und Teilnahmslos-Distanzierte). Milieus, die diesen beiden Gruppen zugeordnet werden können, sind durch ein sehr geringes Vertrauen in das Handeln der politischen Elite charakterisiert. Sie unterscheiden sich allerdings wiederum darin, für wie politisch kompetent sie sich selbst halten. Milieus innerhalb der Gruppe mit der insgesamt grössten Distanz zur Politik, die Teilnahmslos-Distanzierten, weisen geringe bis sehr geringe Werte bei der internen Dimension der politischen Selbstwirksamkeit auf, bei der Gruppe der Entfremdeten Demokratiekritiker schätzen sich Milieus selbst hingegen als politisch kompetent ein. Entsprechend der Kombinationsmöglichkeiten hinsichtlich der beiden richtungspolitischen Konfliktpositionen (libertär-sozialstaatlich, libertärmarktliberal, autoritär-marktliberal, autoritär-sozialstaatlich) erkennen wir auf der horizontal-segmentierenden Dimension wiederum vier Gruppen, denen die einzelnen Milieus zugeordnet werden können. Die «stratamentation»-Heuristik ist insgesamt sehr hilfreich, um die grundlegende Ausdifferenzierung der Bevölkerung in heterogene, politisch-kommunikative Milieus abzubilden. Weitergehend kennzeichnen sich die innerhalb des zweidimensionalen Rasters verorteten Milieus durch jeweils spezifische Merkmale in den 
Abbildung 2: $\quad$ Typologie politisch-kommunikativer Milieus in Deutschland

\begin{tabular}{|c|c|c|c|c|c|}
\hline \multirow{5}{*}{ Nähe zur Politik } & $\begin{array}{l}\text { Aktive Staatsbürger- } \\
\text { schaft } \\
(23 \%)\end{array}$ & $\begin{array}{l}\text { Kritisch } \\
\text { Engagierte } \\
(6 \%)\end{array}$ & $\begin{array}{l}\text { Marktorientiert- } \\
\text { involvierte } \\
(10 \%)\end{array}$ & $\begin{array}{l}\text { Engagierte } \\
\text { Konservative } \\
(7 \%)\end{array}$ & $\begin{array}{l}\text { Engagierte } \\
\text { Konservative } \\
(7 \%)\end{array}$ \\
\hline & & $\begin{array}{l}\text { Sozialdemokrati- } \\
\text { sche Mitte (12\%) }\end{array}$ & & $\begin{array}{l}\text { Gemässigt } \\
\text { Markt-Autoritäre } \\
(10 \%)\end{array}$ & $\begin{array}{l}\text { Autoritäre Mitte } \\
(10 \%)\end{array}$ \\
\hline & $\begin{array}{l}\text { Skeptische Mitte } \\
(19 \%)\end{array}$ & $\begin{array}{l}\text { Unzufriedene } \\
\text { Linke }(13 \%)\end{array}$ & $\begin{array}{l}\text { Staatsskeptische } \\
\text { Individualisten } \\
(6 \%)\end{array}$ & & \\
\hline & $\begin{array}{l}\text { Entfremdete } \\
\text { Demokratiekritiker } \\
(12 \%)\end{array}$ & & & $\begin{array}{l}\text { Konkurrenz-orien- } \\
\text { tierte Rechte } \\
(8 \%)\end{array}$ & $\begin{array}{l}\text { Sozial- } \\
\text { autoritäre } \\
(4 \%)\end{array}$ \\
\hline & $\begin{array}{l}\text { Teilnahmslos- } \\
\text { Distanzierte } \\
(15 \%)\end{array}$ & $\begin{array}{l}\text { Prekär-Distanz- } \\
\text { ierte }(3 \%)\end{array}$ & & $\begin{array}{l}\text { Wenig } \\
\text { Interessierte } \\
(12 \%)\end{array}$ & \\
\hline
\end{tabular}

sonstigen Beschreibungsdimensionen. Über verschiedene Items wurden die Dimensionen «Politische Verortung», «Bezug zur politischen Sphäre», «Kommunikationspraxis», «Sozialer Ort» sowie «Themenrelevanzen» erfasst. Diese können herangezogen werden, um den Zusammenhang zwischen milieuspezifischer Lebenswelt und Deutungen des Flüchtlingsthemas zu erläutern (siehe für eine Übersicht Tabelle 4 und vgl. für eine ausführliche Beschreibung der genutzten Items sowie allen Mittelwerten und Standardabweichungen pro Milieu Kösters \& Jandura, 2018). ((Abb2))

\subsection{Deutungen innerhalb der verschiedenen Milieus}

Mit Bezug zur ersten Forschungsfrage (FF1) erbringt die Analyse der Antworten zum Assoziationstest durch die Befragten sieben verschiedene Deutungen (Fakto- ren) auf das Thema (siehe Tabelle 2). Mit einem Anteil von etwas mehr als 30\% an allen getätigten Aussagen wird die empathisch-offene Perspektive am häufigsten genannt (Tabelle 3, Nennungshäufigkeiten der Themendeutungen). Das Thema Flüchtlingskrise wurde hier vor allem positiv attribuiert, eine Empathie mit den Flüchtlingen wird deutlich und gleichzeitig kommen Befürchtungen gegenüber einer steigenden Fremdenfeindlichkeit zum Ausdruck. Hinter dieser Perspektive verbergen sich Aussagen wie beispielsweise «Wir sollten offen sein für Neues», «Man sollte jedem helfen, der Hilfe braucht» oder "Ich denke an Rassismus und rassistische Diskurse, die in den Diskussionen um geflüchtete Menschen stark bedient werden». An zweiter Stelle wird die Flüchtlingskrise mit den Themendeutungen der nationalistisch-ausgrenzenden Perspekti- 
ve und der medialen Repräsentations-Perspektive in Zusammenhang gebracht. Die Begrenzung der Zuwanderung, ein ausgeprägter Nationalismus sowie die Befürchtung negativer Folgen wie Terrorismus werden dabei mit dem Thema verbunden. Dazu zählen Schlagworte wie «Überfremdung und Kulturverlust» sowie knappe Aussagen wie «Das muss gestoppt werden, wir haben schon genug Probleme» oder auch «Wir holen uns die Terroristen ins Land». Bei der medialen Repräsentations-Perspektive stehen überwiegend kritische Aussagen zum medialen Umgang mit der Flüchtlingskrise mit Aussagen wie «Das ist ein viel zu großer Hype durch die Medien» oder "Man sollte in den Medien stattdessen die Vorteile der Zuwanderung stärker herausstellen» im Vordergrund.

Des Weiteren werden mit einer Gesamthäufigkeit von etwas mehr als sieben Prozent eine elitenkritische Perspektive, in der sich Aussagen zum Umgang, zu Entscheidungen und zum Handeln politischer Eliten wiederfinden, sowie die Chancenperspektive thematisiert. Im Rahmen letzterer werden die aus ökonomischer Sicht positiven Folgen einer steigenden Migration für Deutschland hervorgehoben.

Orientiert man sich an der Rangfolge des Anteils getätigter Aussagen folgt an vorletzter Stelle die gemässigt rechtpopulistische Perspektive (fast sechs Prozent der Nennungen). Diese vereint ebenfalls negative Deutungen des Themas. Hier werden Sorgen um eine steigende Kriminalität und rückläufige Sicherheit ebenso geäussert wie negative sozioökonomische Konsequenzen des Zuzugs von Migranten. Abschliessend wird die Perspektive diffuser negativer Folgen (4\%), wie Terrorismus und wachsender Fremdenfeindlichkeit, genannt.

Die Aufzählung zeigt, dass die Deutungen in der vorliegenden Studie sehr differenziert sind und sich nicht nur auf eine Dichotomie von positiven und negativen Positionen reduzieren lassen. Ferner sind sie mit wertebezogenen, politisch-kulturellen und sozioökonomischen Konfliktpositionen verbunden.

Im Folgenden wird ausgeleuchtet, ob bestimmte Deutungen der Flüchtlingskri- se in bestimmten politisch-kommunikativen Milieus anzutreffen sind oder ob diese gleich über die Bevölkerung verteilt sind (siehe Tabelle 3; FF2). Eine homogene milieuspezifische Sichtweise würde dabei als gesellschaftlich potenziell desintegrativ, eine hohe Vielfalt der Deutungen innerhalb eines Milieus hingegen als potenziell integrativ bewertet werden. Aus einer hohen Vielfalt leiten wir einerseits ab, dass das jeweilige Milieu selbst unterschiedliche Ansichten in sich vereint, und andererseits, dass innerhalb des Milieus eine Offenheit gegenüber anderen Perspektiven herrscht.

Für einen Vergleich der Pluralität der Deutungen von Milieus sind in Tabelle 3 drei Kennwerte eingetragen. Zum einen wird bei allen Themendeutungen zusätzlich zu den prozentuellen Anteilen in zweiter Spalte auch ein Vergleichswert (Differenz der relativen prozentuellen Anteile der Nennungen einer Perspektive im jeweiligen Milieu zum Anteil der Nennungen der betrachteten Perspektive über alle Milieus hinweg) angegeben. Zum anderen wird die Perspektivenvielfalt (letzte Spalte ganz rechts) durch ein Konzentrationsmass der (Un-)Gleichheit der Verteilung der Antworten (modifizierter Herfindahl-Index) abgebildet. Von der zuvor beschriebenen desintegrativen Tendenz würden wir dann ausgehen, wenn sich zeigt, dass bestimmte Milieus überproportional häufig eine oder nur einige wenige Deutungen einnehmen und das Mass zur Erfassung der Vielfalt der Perspektiven entsprechend höhere Werte annimmt. Zusammenfassend nimmt das angegebene Mass der Perspektivenvielfalt im Milieuvergleich Werte zwischen 0,19 und 0,24 an, was sich zunächst als Anzeichen dafür interpretieren lässt, dass die Milieus hinsichtlich der Themendeutungen keine vollständig sozial und politisch abgeschotteten Einheiten darstellen. So findet beispielsweise auch in ein Milieu wie die Sozialdemokratische Mitte die nationalistisch-ausgrenzende Perspektive Eingang. Besonders auffällig ist im Hinblick auf das Mass zur Perspektivenvielfalt $(0,246)$ das Milieu der Wenig Interessierten, die mit einem Anteil von fast 34 Prozent überpro- 
portional häufig eine nationalistisch-ausgrenzende Perspektive einnehmen und bei allen anderen Themendeutungen deutlich weniger Aussagen getätigt haben. ${ }^{6}$

Aus dem Überblick über die milieuspezifischen Verteilungen der Deutungen kann keine gruppenübergreifende Polarisierung der Deutungen abgeleitet werden, dennoch zeigen sich je nach $\mathrm{Mi}-$ lieu sowohl desintegrative als auch integrative Tendenzen. Wir finden auf Grundlage der verschiedenen Kennzahlen etwa eine Reihe von Milieus, in denen eine der identifizierten Hauptperspektiven (empathisch-offene sowie nationalistisch-ausgrenzende Perspektive) dominant ist. Dies deutet auf eine eher desintegrative Richtung hin.

So entfallen gemäss der in Tabelle 3 dargestellten Gesamthäufigkeiten etwa $35 \%$ der Aussagen des Milieus der Sozialdemokratischen Mitte auf die empathisch-offene Perspektive. Antworten, die zur nationalistisch-ausgrenzenden Perspektive oder zur damit verwandten gemässigt rechtspopulistischen Perspektive gezählt werden können, sind hingegen zusammengenommen in ca. $22 \%$ der Fälle zu finden. Eine ähnliche Verteilung liegt bei den Kritisch-Engagierten vor. Hier entfallen 38\% der Antworten auf die empathisch-offene Perspektive, während knapp über $20 \%$ der Antworten auf beide rechtspopulistische Perspektiven zurückzuführen sind. Letztgenannte Perspektiven treten hingegen insbesondere bei den Milieus der Autoritären Mitte (55\% zu 15\%), den Wenig Interessierten (45\% zu 9\%), den Gemässigt Markt-Autoritären ( $40 \%$ zu nur knapp über 0\%), den Sozialautoritären (62\% zu $26 \%$ ) sowie den Konkurrenzorientierten Rechten (57\% zu 23\%) dominant auf.

6 Neben dem Milieu der Wenig Interessierten sind es mit Blick auf das Konzentrationsmass zur Perspektivenvielfalt die Prekär-Distanzierten, die einen besonders auffälligen Konzentrationswert tragen. Bei genau diesen beiden Milieus handelt es sich um gesellschaftliche Teilgruppen, die durch Abkopplungstendenzen vom öffentlichen Diskurs charakterisiert sind (siehe weiterführend Kösters \& Jandura, 2018).
Dass etwa bei den Sozialautoritären und den Konkurrenzorientierten Rechten (oder andererseits auch bei richtungspolitisch linken Milieus wie den Kritisch-Engagierten oder der Sozialdemokratischen Mitte, siehe oben) eine inhaltlich konträre Deutung relativ gesehen ebenfalls prominent ist, liesse sich über die Valenz erklären mit der Befragte entsprechende Assoziationen zum Ausdruck bringen. Das hiesse etwa, dass im Milieu der Konkurrenzorientierten Rechten Assoziationen genannt werden, die für eine Empathie mit der Situation von Flüchtlingen stehen, diese aber wiederum negativ bewertet werden. Während die Perspektiven-spezifische Valenz im Rahmen der Studie nicht empirisch erfasst wurde, so kann die Abfrage der generellen Bewertung des Themas «Zuwanderung von Flüchtlingen» in diesem Zusammenhang zumindest erste Hinweise geben. Für die Sozialautoritären und Konkurrenzorientierten Rechten zeigt sich nämlich beispielsweise eine vergleichsweise extrem negative, allgemeine Bewertung des Themas (siehe Tabelle $5 \mathrm{im}$ Anhang).

Besonders bei den hinsichtlich ihrer politischen Ausrichtung her eher extremen Milieus der Konkurrenzorientierten Rechten und den Sozialautoritären ist die insgesamt einseitige Deutung in demokratietheoretischer Hinsicht als desintegrativ zu bewerten, da bei beiden Milieus in ihrem politischen Kommunikationsverhalten sowohl bei der Mediennutzung, als auch bei der interpersonalen Kommunikation zusätzlich Abschottungstendenzen zu beobachten sind, die zu einer Polarisierung der Gesellschaft führen können. Hinweise für solche Tendenzen lassen sich einerseits aus den Informationsrepertoires der Milieus ableiten, in denen Tageszeitungen der politischen Extrema, politische Blogs sowie soziale Netzwerke von hoher Bedeutung sind. Andererseits ist die politische Homogenität der Kommunikationsnetzwerke dieser Milieus ein Indikator dafür, dass diese Milieus im interpersonalen Austausch kaum mit anderen Sichtweisen in Kontakt kommen (siehe weiterführend dazu Kösters \& Jandura, 2018). 


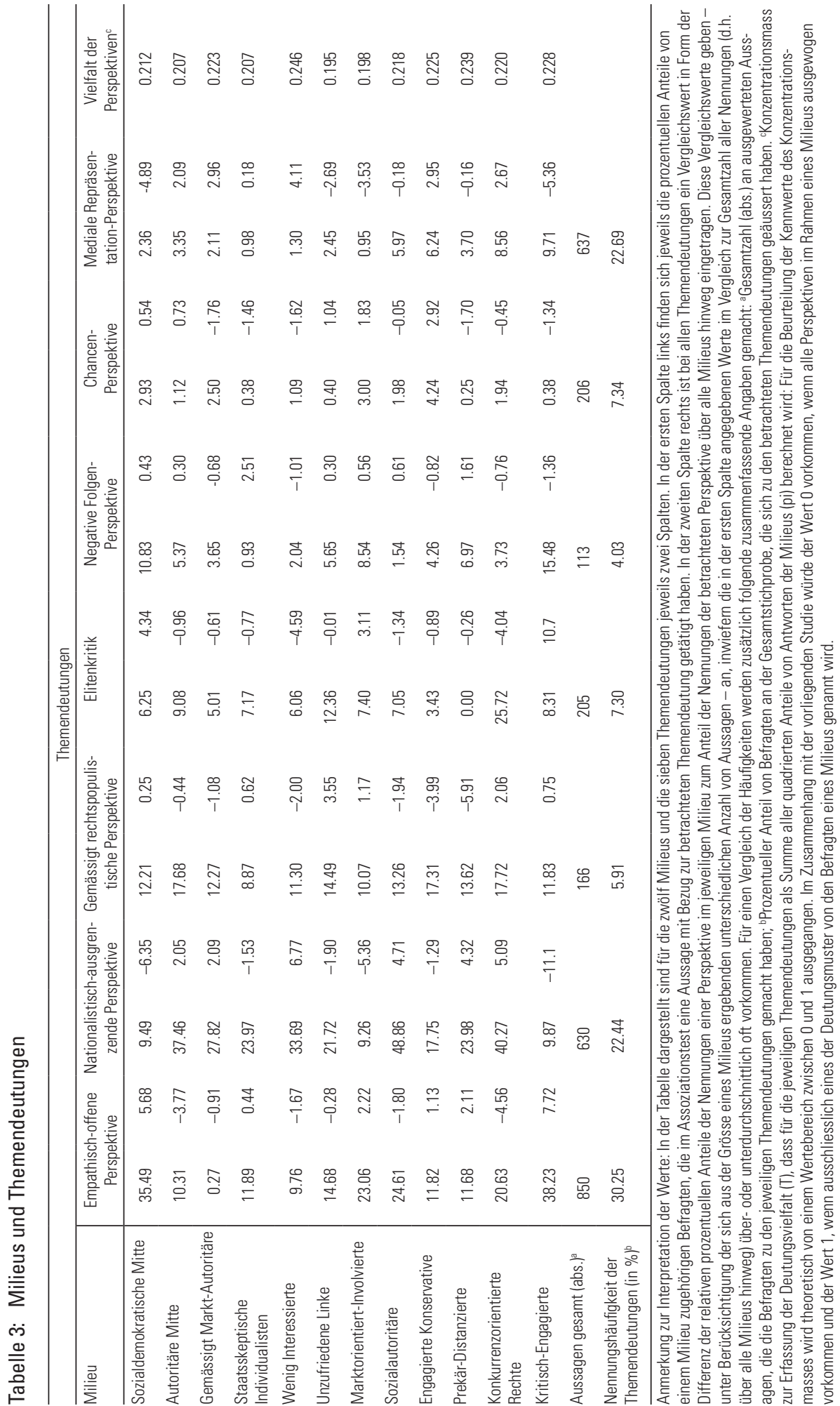


In anderen Milieus ist eine grössere Pluralität der Deutungen der Flüchtlingskrise $\mathrm{zu}$ verzeichnen. Als herausragendes Beispiel ist hier das Milieu der Unzufriedenen Linken zu nennen. Hier sind die empathisch-offene Perspektive (15\%), die nationalistisch-ausgrenzende Perspektive (22\%) sowie die gemässigt rechtspopulistische Perspektive (14\%) ungefähr gleichverteilt. Zudem wird das Thema häufig mit der Elitenkritik (12\%) in Verbindung gebracht. Ähnlich sieht es etwa bei den Staatsskeptischen Individualisten und den Marktorientiert-Involvierten aus. Obwohl hier eine der dominanten Perspektiven relativ gesehen am häufigsten genannt wird, finden sich innerhalb der Milieus dennoch auch andere Deutungen mit bemerkenswerten Anteilen (vgl. dazu etwa auch den Kennwert derVielfalt der Perspektiven, der bei diesen Milieus kleiner oder ungefähr gleich 0,2 ist). Dies könnte ein Hinweis darauf sein, dass die Wahrscheinlichkeit mit anderen Themendeutungen in den milieuspezifischen politischen Teilöffentlichkeiten konfrontiert zu werden, für die Bürger, die diesen Milieus zugehörig sind, grösser ist. Dazu passt, dass sich für diese Milieus - anders als bei den Sozialautoritären oder den Konkurrenzorientierten Rechten - etwa keine ausgeprägte politische Homogenität der interpersonalen Kommunikationsnetzwerke ausmachen lässt (Kösters \& Jandura, 2018). Insgesamt wäre die Grundvoraussetzung für den Austausch zwischen den Bürgern und die Wahrnehmung von unterschiedlichen Deutungen zur Flüchtlingskrise sowie die Verortung der Positionen des Einzelnen im öffentlichen Raum hier eher gegeben.

In einem weiteren Schritt sollen nun die Deutungen der Flüchtlingsthematik mit der milieuspezifischen Lebenswelt in Verbindung gebracht werden (FF3). Hier-

Tabelle 4: Charakteristika der Milieus «Sozialdemokratische Mitte» und «Unzufriedene Linke»

\begin{tabular}{|c|c|c|}
\hline Beschreibungsdimension & Sozialdemokratische Mitte & Unzufriedene Linke \\
\hline $\begin{array}{l}\text { Politische Verortung } \\
\text {-Wertorientierungen, } \\
\text {-Parteipräferenz, } \\
\text {-Differenzsemantiken }\end{array}$ & $\begin{array}{l}\text { * eher sozialstaatlich } \\
\text { * libertär } \\
\text { * Parteipräferenz: Grüne (63\%), SPD (56\%) } \\
\text { * SPD im Gesamtvergleich am stärksten } \\
\text { * hoch ausgeprägte Sorge um Betrachtung } \\
\text { von Ausländern als Belastung }\end{array}$ & $\begin{array}{l}\text { * sozialstaatlich } \\
\text { * libertär } \\
\text { * Parteipräferenz: Linke (40\%), Grüne (39\%), } \\
\text { SPD }(30 \%) \text { - relativ geringe Ausprägungen } \\
\text { für AfD sowie bürgerliches Lager } \\
\text { * ausgeprägte Kritik an Entscheidungskompe- } \\
\text { tenz der EU, ausgeprägte Elitenkritik }\end{array}$ \\
\hline $\begin{array}{l}\text { Bezug zur politischen Sphäre } \\
\text {-Interne \& externe pol. Selbstwirksamkeit, } \\
\text { pol. Interesse, mediales Repräsentations- } \\
\text { gefühl, politische Partizipation, Demokratie- } \\
\text { zufriedenheit }\end{array}$ & $\begin{array}{l}\text { * interne Efficacy unterdurchschnittlich } \\
{ }^{*} \text { hohe externe Efficacy }\end{array}$ & $\begin{array}{l}\text { * durchschnittliche interne Efficacy } \\
\text { * sehr geringe externe Efficacy } \\
\text { * relativ geringe Demokratiezufriedenheit }\end{array}$ \\
\hline $\begin{array}{l}\text { Sozialer Ort } \\
\text {-Geschlecht, Alter, Bildung, Beruf, Einkom- } \\
\text { men, Herkunft, Lebenszufriedenheit }\end{array}$ & $\begin{array}{l}\text { * eher jung, tendenziell höherer Bildungsgrad, } \\
\text { relativ viele Auszubildende und vergleichs- } \\
\text { weise wenig Rentner, tendenziell etwas } \\
\text { geringere Einkommen }\end{array}$ & $\begin{array}{l}\text { * relativ viele Nicht-Erwerbstätige/Arbeit- } \\
\text { slose, sofern berufstätig ist die begrenzte } \\
\text { Verantwortung für Tätigkeit anderer relativ } \\
\text { häufig, eher niedrige Einkommen, Lebenszu- } \\
\text { friedenheit sehr niedrig }\end{array}$ \\
\hline $\begin{array}{l}\text { Themenrelevanz } \\
\text {-Top } 5 \text { der genannten Einzelthemen }\end{array}$ & $\begin{array}{l}\text { 1. Zuwanderung } \\
\text { 2. Rente } \\
\text { 3. Freihandelsabkommen } \\
\text { 4. Bildungspolitik } \\
\text { 5. Verteilungsgerechtigkeit }\end{array}$ & $\begin{array}{l}\text { 1. Zuwanderung } \\
\text { 2. Rente } \\
\text { 3. Altersarmut, Verteilungsgerechtigkeit } \\
\text { 4. Freihandelsabkommen, Bildungspolitik } \\
\text { 5. Agenda 2010, Hartz IV }\end{array}$ \\
\hline
\end{tabular}

Anmerkung: Aufgeführt sind Charakteristika, bei denen sich die jeweiligen Milieus hinsichtlich der Mittelwerte in den betrachteten Variablen von allen anderen Milieus unterscheiden. 
für greifen wir mit den Milieus der Unzufriedenen Linken und der Sozialdemokratischen Mitte exemplarisch zwei Milieus heraus, die sich in ihrer politischen Verortung eher ähnlich sind, bei denen aber einerseits eine plurale und andererseits eine dominant positiv konnotierte Deutung der Flüchtlingskrise $\mathrm{zu}$ verzeichnen ist. Die für beide Milieus charakterisierenden Eigenschaften sind in Tabelle 4 aufgeführt.

Die politische Grundorientierung beider Milieus lässt sich bei der sozialstaatlichen Position auf der sozioökonomischen Konfliktdimension und der liberalen Position auf der politisch-kulturellen Konfliktdimension festmachen. Unterschiede finden sich jedoch auf der stratifikatorischen Dimension. Während im Milieu der Unzufriedenen Linken ein durchschnittliches politisches Kompetenzgefühl, aber ein sehr geringes Responsivitätsvertrauen $\mathrm{zu}$ finden ist, zeigt sich bei der Sozialdemokratischen Mitte ein eher unterdurchschnittliches Kompetenzgefühl aber ein hohes politisches Responsivitätsvertrauen. Diese unterschiedliche Nähe zum politischen System geht bei der erstgenannten Gruppe mit einer sehr geringen Demokratiezufriedenheit und bei der Sozialdemokratischen Mitte mit einer eher hohen Demokratiezufriedenheit einher. Trotz einer gemeinsamen Präferenz für die linken Parteien SPD, Grüne und Linke unterscheiden sich die Milieus in ihren Einschätzungen der sozialen Differenzsemantiken. ${ }^{7}$ Während die Sozialdemokratische Mitte eher kosmopolitisch eingestellt ist und sich für eine Integration von Migranten einsetzt, finden wir bei der Unzufriedenen Linken eine eher auf das Nationale ausgerichtete Position mit Tendenzen zur Ausgrenzung von Fremden und Elitenkritik.

In ihrem sozialstrukturellen Hintergrund unterscheiden sich beide Milieus sehr deutlich. Die soziale Verortung politisch-kommunikativer Milieus wird - da-

7 Auf Kurzformeln gebracht wurden die folgenden Differenzsemantiken über jeweils zwei Items abgefragt und in der Analyse näher betrachtet: «oben vs. unten», «eigen vs. Fremd» sowie «Nation vs. supranationale Kooperation». durch, dass sie mit differenten sozialen Erfahrungskontexten einhergeht - als ein entscheidender Faktor für die Ausbildung von politischen Orientierungen und Grundhaltungen gegenüber der politischen Sphäre (politisches Interesse, politische Selbstwirksamkeit) erachtet (Kitschelt, 1994; Weiß, 2009). Das Milieu der Unzufriedenen Linken ist im unteren Bereich des sozialen Raums zu lokalisieren. Nicht-Erwerbstätigkeit und untere Einkommen dominieren das Bild. Die Lebenszufriedenheit ist eher niedrig. Die soziale Position des Milieus der Sozialdemokratischen Mitte lässt sich hingegen nicht klar bestimmen, da neben gesellschaftlich etablierten Milieuzugehörigen sich viele noch in der Ausbildungsphase beziehungsweise der Phase des Berufseinstiegs befinden. Hier finden wir auch eher niedrige Einkommen, jedoch einen vergleichsweise höheren Bildungsgrad und eine höhere Lebenszufriedenheit. Die soziale Lage spiegelt sich auch in den Themenrelevanzen der Milieus wieder. Nach den Top-Themen Zuwanderung und Rente ist für das Milieu der Unzufriedenen Linken Altersarmut und Verteilungsgerechtigkeit relevant, während das Freihandelsabkommen und die Bildungspolitik für die Sozialdemokratische Mitte wichtig sind.

Die pro Milieu jeweils im sozialen Ort verankerte Verbindung einer sozialstaatlichen und libertären Position bei unterschiedlichem Responsivitätsvertrauen und bei differierenden Einstellungen zu soziologischen Differenzsemantiken geht einher mit einer sich stark unterscheidenden Deutung des Themas Flüchtlingskrise. Während bei der Sozialdemokratischen Mitte die empathisch-offene Perspektive auf das Thema mit 35\% der Nennungen dominant ist und eine nationalistisch-ausgrenzende Perspektive mit 9\% nur selten bedient wird, sind beide Deutungen bei den Unzufriedenen Linken eher gleichverteilt ( $15 \%$ zu $21 \%$ ). Passend zum geringen Responsivitätsvertrauen des letztgenannten Milieus findet sich ein hoher Anteil der Perspektive der Elitenkritik (15\%). Bei dieser vergleichenden Betrachtung der beiden Milieus wird die, u.a. von Grande und Kriesi (2012) für politische Parteien 
beschriebene Spaltung in eine «neue» und eine «alte» Linke hinsichtlich der Themenfelder Integration und Immigration - hier allerdings mit Blick auf die Bevölkerung sehr deutlich.

Eine wichtige, ohne nähere Analysen an dieser Stelle jedoch nicht zu klärende Frage bleibt, in welchem Verhältnis die Kommunikationspraxis der beiden näher betrachteten Milieus und die daraus resultierende Rezeption und Verarbeitung bestimmter Medieninhalte $\mathrm{zu}$ den grundsätzlichen Politikeinstellungen und themenspezifischen Deutungen stehen (siehe Diskussion unten). Auch hinsichtlich der Kommunikationspraxis zeigen sich nämlich Differenzen. Im Milieu der Sozialdemokratischen Mitte prägen eindeutig Hard News (öffentlich-rechtliche Fernsehnachrichten und - auf Basis der Befragungsdaten nicht näher zu bestimmende - Online-Qualitätszeitungen) die politische Mediennutzung. Bei den Unzufriedenen Linken zeigt sich hingegen eine leicht unterdurchschnittliche Nutzung von Informationsangeboten mit Ausnahme von Boulevardzeitungen.

\section{Fazit und Diskussion}

Theoretischer Ausgangspunkt unseres Beitrags war eine Kritik am gebräuchlichen Zugang der Fragmentierungsforschung, über die empirische Identifikation von gesellschaftsweit geteilten Themen auf der Bevölkerungsagenda, Rückschlüsse auf eine integrative Wirkung zu ziehen. Mit Blick auf Erkenntnisse aus Forschungsfeldern, die sich einerseits mit Medienagenden und andererseits mit der Verarbeitung medialer Inhalte seitens des Publikums auseinandersetzen, kann die Annahme formuliert werden, dass hinter geteilten Themen - die es trotz Fragmentierungstendenzen weiterhin gibt - ganz unterschiedliche Deutungen stehen. Dies mag intuitiv einleuchten, es geht aber vor allem darum zu bekräftigen, dass diese Deutungen zusätzlich in den Blick genommen werden sollten, um Aussagen über das integrative Potenzial gemeinsamer Themen treffen zu können. Zur Verdeutlichung unseres theoretischen Arguments haben wir eine nähere Analyse zu einem Thema durchgeführt, das seit geraumer Zeit ganz oben auf der Agenda heterogener Milieus der Gesellschaft steht - Migration bzw. spezifischer die Zuwanderung von Flüchtlingen.

Insgesamt zeigt unsere Untersuchung der bevölkerungsseitigen Deutungen zur so bezeichneten "Flüchtlingskrise», dass das untersuchte Thema zwar in allen Milieus als wichtig identifiziert wird, sich auf der Ebene der Deutungen aber zum Teil fundamental andere Interpretationen gegenüberstehen. Die jeweiligen Perspektiven sind gemäss einer grundsätzlichen Annahme des milieutheoretischen Zugangs massgeblich durch den lebensweltlichen Kontext einzelner Bevölkerungsgruppen geprägt. Dies wurde exemplarisch anhand einer näheren Betrachtung von zwei Milieus (Sozialdemokratische Mitte und Unzufriedene Linke) erläutert. Zudem unterscheiden sich die Milieus danach, ob einzelne Deutungen zum Thema jeweils sehr dominant sind oder ob innerhalb desselben Bevölkerungssegments verschiedenste Sichtweisen vorkommen. Öffentlichkeitstheoretisch problematisch erscheinen jene politisch vergleichsweise extremen Milieus, in denen nicht nur bestimmte Sichtweisen dominant sind, sondern die sich - mit Blick auf ihre mediale und interpersonale Kommunikationspraxis - durch Tendenzen zur Abschottung kennzeichnen. Die Befunde zu Milieus mit extremen Grundhaltungen sprechen trotz des geteilten Themas - gegen eine integrative und eher für eine polarisierende Wirkung der Debatte. Demgegenüber stehen Milieus, aus deren Antwortverhalten zu unserem «Assoziationstest» zwar ebenfalls eine Präferenz für bestimmte Deutungen zu erkennen ist, in denen aber auch andere Perspektiven präsent sind und deren Kommunikationsnetzwerke sich nicht durch eine deutliche politische Homogenität auszeichnen. Für diese Milieus ist prinzipiell ein integrierendes Potenzial des gemeinsamen Themas abzuleiten. Die Untersuchung zeigt konzeptionell, dass die Betrachtung von Themendeutungen relevant ist, um das integrierende Potenzi- 
al gemeinsamer Themen besser einschätzen zu können. Es ist keineswegs ausreichend, gemeinsame Themen zwischen Segmenten der Bevölkerung auszumachen. Es wird darüber hinaus eine weitere Analyseebene benötigt, jene der Deutungen. Der Zugang über den Milieu-Ansatz bietet insgesamt die Möglichkeit, differenzierte Aussagen darüber zu treffen, welche Haltungen wie zu erklären sind und in welchen Teilen der Bevölkerung Chancen auf eine die Gesellschaft vernetzende Kommunikation (noch) gegeben sind.

Aus methodischer Sicht hat die Einbindung eines offenen Assoziationstestes in die standardisierte Online-Befragung den erhofften Nutzen gebracht, d.h. wir konnten durch den Verzicht auf vorgegebene Auswahloptionen eine Bandbreite von themenbezogenen Sichtweisen in der Bevölkerung erfassen. Dies bezieht sich nicht nur darauf, dass je nach $\mathrm{Mi}$ lieu unterschiedliche Themenaspekte hervorgehoben wurden, sondern meint auch, dass Unterschiede im Differenziertheitsgrad der Antworten deutlich wurden (Differenzierung von mehr oder weniger elaborierten Antworttypen). Dennoch sind dem methodischen Verfahren auch Grenzen gesetzt. Gerade bei Milieus, die etwa aufgrund von fehlendem politischen Grundwissen weniger in der Lage sind, ihre Ansichten zu artikulieren, scheinen qualitative Interviews besser geeignet $\mathrm{zu}$ sein, um einen besseren Einblick in die jeweilige politische Weltsicht zu erlangen. Eine weitere Möglichkeit für Anschlussstudien wäre es, auf Grundlage unserer explorativen Studie und den so ermittelten Faktoren und Antworten, Items für geschlossene, standardisierte Befragungen abzuleiten. Auch über diesen Zugang mag es für bestimmte Milieus im Vergleich $\mathrm{zu}$ einer offenen Fragestellung einfacher zu sein, ihre Sichtweisen auszudrücken. Zudem könnten im Sinne einer theoretisch-konzeptionellen Erweiterung Erwartungen gegenüber den Antwortmustern bestimmter Milieus formuliert und die standardisierten Befragungsdaten dann anhand einer konfirmatorischen Faktorenanalyse untersucht werden. In unserer Studie ging es vornehmlich darum, zu- nächst die Verschiedenheit der Themenperspektiven zu identifizieren. Zusätzlich erachten wir es aber als gewinnbringend, in Folgestudien auch die Valenz der Perspektiven zu erfassen. So könnten die milieuspezifischen Begründungen einer ablehnenden oder zustimmenden Haltung zum Thema näher untersucht werden. Die Berücksichtigung der Valenz könnte auch dabei helfen, Tendenzen einer über die Fragmentierung hinausgehenden Polarisierung von Themenperspektiven differenziert $\mathrm{zu}$ erfassen. Beispielsweise könnte geprüft werden, ob in bestimmten Gesellschaftssegmenten konträre Themendeutungen nur deshalb aufgegriffen werden, um diese wiederum abzuwerten.

Hinsichtlich möglicher Folgestudien möchten wir uns ferner der Forderung anschliessen, dass die Forschung zukünftig verstärkt die verschiedenen Analyseebenen von Fragmentierung (Angebote, Inhalte, Publikum und Nutzung sowie Rezeption und Wirkung) miteinander verknüpfen sollte (u.a. Kleinen-von Königslöw, 2016). Im Hinblick auf die vorliegende Studie bietet es sich beispielsweise an, themenspezifische Medieninhaltsanalysen für relevante Angebote aus den Informationsrepertoires der einzelnen betrachteten Milieus durchzuführen, um die öffentlichkeitstheoretisch wünschenswerte Pluralität der Perspektiven in den Medieninhalten mit den lebensweltlich begründeten Problemsichten der jeweiligen Rezipienten $\mathrm{zu}$ vergleichen (Verbindung der Inhalts- und Publikums-/Nutzungsebene).$^{8}$ Ohne tatsächliche Wirkungen $\mathrm{zu}$ messen, liessen sich darüber bereits weiterführende Interpretationen zum $\mathrm{Zu}$ sammenhang der lebensweltlich eingebetteten Kommunikationspraxis und milieuspezifischen Problemdeutungen zum fokussierten (Krisen-)Thema sowie zum mehr oder weniger integrativen, womöglich sogar eher polarisierenden Leistungsvermögen verschiedener Medientypen

8 Konzeptionell könnten hier Framing-Studien (z. B. Budde et al., 2018) oder Analysen zur medialen Vermittlung von normativen Grundhaltungen (Value-Framing; Weiß \& Jandura, 2017; Weiß et al., 2016) angewandt werden. 
ableiten. Über eine solche Herangehensweise liesse sich das Wechselspiel zwischen einem publizistischen Konflikt und dessen Resonanz auf Bevölkerungsseite näher beleuchten.

Gesellschaftliche Krisen berühren zwar grosse Teile einer Gesellschaft in ähnlicher Weise und sind damit geradezu prädestiniert, zu einem gemeinsam geteilten Thema zu werden. Aufgrund ihres bisweilen polarisierenden Charakters können sie allerdings eher eine Herausforderung für den gesellschaftlichen Zusammenhalt darstellen. Übertragen auf die Fragmentierungsdebatte führt diese Beobachtung $\mathrm{zu}$ der Frage, ob eine gesellschaftlich geteilte Themenbasis als hinreichende Bedingung immer integrativ wirkt. Und falls dies eben nicht so ist, welche weiteren Voraussetzungen es für die Integrationskraft gemeinsamer Themen gibt. Dazu könnte zukünftig die Beschaffenheit von Themen stärker in den Blick genommen werden. Hierzu lohnt der Rückgriff auf in der Politikwissenschaft vorliegende Issue-Typologien. Geläufig ist hier beispielsweise die Unterscheidung von hinsichtlich der angestrebten normativen Zielvorstellungen wenig kontroversen Valenzissues (z. B. Arbeitslosigkeit, Bekämpfung von Korruption) und Positionsissues, die deshalb stärker umstritten sind, weil die dazu existierenden Positionen wiederum gegensätzliche Zielvorstellungen ansprechen (Stokes, 1963; siehe weiterführend auch Green, 2007; Guinaudeau \& Persico, 2013). Van Hoof und Kolleginnen (2014) unterscheiden weiter zwischen Themen, die z.B. «typisch progressiv» (Bürgerrechte) oder «typisch konservativ» (Durchsetzung von Recht und Ordnung) sind, sowie Konsensthemen, die in etwa den genannten Valenzissues entsprechen. Das integrative Potenzial von gemeinsamen Themen auf Ebene der Bevölkerung könnte sich ferner danach unterscheiden, wie stark umstritten Themen auf Seiten der politischen und medialen Elite sind (Bennett, 2016). Wünschenswert wären in diesem Zusammenhang Anschlussstudien, die ein ähnliches Vorgehen wie der vorliegende Beitrag wählen, dabei aber die bevölkerungsseitigen Deutungen zu gemeinsamen, hinsichtlich ihrer Beschaffenheit jedoch voneinander abweichenden Themen miteinander vergleichen.

\section{Literatur}

Arlt, D. \& Wolling, J. (2017). Die Flüchtlingsdebatte in den Medien aus der Perspektive der Bevölkerung. Veränderungen von Nutzungsmustern, Erwartungen, Bewertungen und Einstellungen zwischen 2016 und 2017. Media Perspektiven, 6, 325-337.

Ball-Rokeach, S. J. \& Loges, W. E. (1996). Making choices. Media roles in the construction of value-choices. In C. Seligman, J. M. Olson, \& M. P. Zanna (Eds.), The psychology of values (The Ontario Symposium, vol. 8 , pp. 277-298). Mahwah and New York: Erlbaum.

Begenat, M. (2016). Öffentlichkeit - für alle? Themen und Informationsrepertoires in politischen Milieus. Wiesbaden: Springer VS.

Begenat, M. (2017). Im Horizont aller? Zur Fragmentierung politischer Kommunikation entlang politischer Milieus. In O. Jandura, M. Wendelin, M. Adolf, \& J. Wimmer (Hrsg.), Zwischen Integration und Diversifikation. Medien und gesellschaftlicher Zusammenhalt im digitalen Zeitalter (S. 189-206). Wiesbaden: Springer VS.

Beierlein, C., Kemper, C. J., Kovaleva, A., \& Rammstedt, B. (2012). Ein Messinstrument zur Erfassung politischer Kompetenz- und Einflussüberzeugungen. Political Efficacy Kurzskala (PEKS), GESIS - Leibniz-Institut für Sozialwissenschaften. GESIS-Working Papers: 18. Zugriff am 15.06.2018. Verfügbar unter https://www.gesis.org/ fileadmin/kurzskalen/working_papers/ PEKS_Workingpaper.pdf

Bennett, L. W., \& Iyengar, S. (2008). A new era of minimal effects? The changing foundations of political communication. Journal of Communication, 58(4), 707-731. doi:10.1111/j.1460-2466.2008.00410

Bennett, L.W. (2016). Indexing theory. In G. Mazzoleni (Hrsg.), The International Encyclopedia of Political Communication. doi:10.1002/9781118541555.wbiepc180

Besova, A. A., \& Cooley, S. C. (2009). Foreign news and public opinion. Attribute agen- 
da-setting theory revisited. Ecquid Novi:

African Journalism Studies, 30(2), 219-242. doi:10.1080/02560054.2009.9653403

Budde, N., Jandura, O., \& Dohle, M. (2018). Das Framing der Flüchtlingskrise in Parlament und Parlamentsmagazinen. Mitteilungen des Instituts für Deutsches und Internationales Parteienrecht und Parteienforschung MIP, 24, 31-39.

Cacioppo, J. T., Harkins, S. G., \& Petty, R. E. (1981). The nature of attitudes and cognitive responses and their relationships to behavior. In R. E. Petty, T. M. Ostrom, \& T. C. Brock (Hrsg.), Cognitive responses in persuasion (pp. 31-54). Hillsdale: Erlbaum.

Eilders, C. (2004). Von Links bis Rechts. Deutung und Meinung in Pressekommentaren. In C. Eilders, F. Neidhardt, \& B. Pfetsch (Hrsg.), Die Stimme der Medien. Pressekommentare und politische Öffentlichkeit in der Bundesrepublik (S. 129-166). Opladen: VS Verlag für Sozialwissenschaften.

Eilders, C. (2013). Öffentliche Meinungsbildung in Online-Umgebungen. Zur Zentralität der normativen Perspektive in der politischen Kommunikationsforschung. In M. Karmasin, M. Rath, \& B. Thomaß (Hrsg.), Normativität in der Kommunikationswissenschaft (S. 329-351). Wiesbaden: VS Verlag für Sozialwissenschaften.

Ferree, M. M., Gamson, W. A., Gerhards, J., \& Rucht, D. (2002). Shaping abortion discourse. Democracy and the public sphere in Germany and the United States. Cambridge: Cambridge University Press.

Gabriel, S. (2016, Mai). Grundsatzrede auf der Wertekonferenz Gerechtigkeit der SPD. Berlin.

Gerhards, J. (1998). Konzeptionen von Öffentlichkeit unter den heutigen Medienbedingungen. In O. Jarren, \& F. Krotz (Hrsg.), Öffentlichkeit unter Viel-KanalBedingungen (S. 25-47). Baden-Baden: Nomos.

Grande, E., \& Kriesi, H. (2012). The transformative power of globalization and the structure of political conflict in Western Europe. In H. Kriesi, E. Grande, M. Dolezal, M. Helbling, D. Höglinger, S. Hutter et al. (Hrsg.), Political conflict in Western Europe (S. 3-35). Cambridge: Cambridge University Press.
Green, J. (2007). When voters and parties agree. Valence issues and party competition. Political Studies, 55(3), 629-655. doi: 10.1111/j.1467-9248.2007.00671.x

Guinaudeau, I., \& Persico, S. (2014). What is issue competition? Conflict, consensus and issue ownership in party competition. Journal of Elections, Public Opinion \& Parties, 24(3), 312-333. doi:10.1080/17457289.2013.858344

Haas, A., \& Brosius, H.-B. (2013). Fragmentierung der Publikumsagenda im Zeitverlauf? In K. Imhof, R. Blum, H. Bonfadelli, \& O. Jarren (Hrsg.), Stratifizierte und segmentierte Öffentlichkeit (S. 187-202). Wiesbaden: Springer VS.

Häusler, A. (Hrsg.). (2016). Die Alternative für Deutschland. Programmatik, Entwicklung und politische Verortung. Wiesbaden: SpringerVS.

Helbling, M. (2014). Framing immigration in Western Europe. Journal of Ethnic and Migration Studies, 40(1), 21-41. doi:10.1080/1369183X.2013.830888

Hradil, S. (2006). Soziale Milieus. Eine praxisorientierte Forschungsperspektive. Aus Politik und Zeitgeschichte, o. Jg. (44-45), 3-10.

Imhof, K. (2011). Die Krise der Öffentlichkeit. Kommunikation und Medien als Faktoren des sozialen Wandels. Frankfurt: Campus.

Imhof, K. (2013). Austritt aus der selbstverschuldeten Unmündigkeit. Wie differenzieren wir das Soziale? In K. Imhof, R. Blum, H. Bonfadelli, \& O. Jarren (Hrsg.), Stratifizierte und segmentierte Öffentlichkeit (S. 79-90). Wiesbaden: Springer VS. infratest dimap. (2015). Deutschland Trend 12/2015. Zugriff am 15.06.2018. Verfügbar unter https:/ /www.infratest-dimap. de/umfragen-analysen/bundesweit/ umfragen/aktuell/54-prozent-derbevoelkerung-meinen-wir-schaffen-esnicht-die-fluechtlinge-erfolgreich-zuintegrieren/

Hall Jamieson, K., \& Cappella, J. N. (2008). Echo chamber. Rush Limbaugh and the conservative media establishment. Oxford: Oxford University Press.

Jarren, O. (2000). Gesellschaftliche Integration durch Medien? Zur Begründung normativer Anforderungen an Medien. Medien und Kommunikationswissenschaft, 48(1), 22-41. doi:10.5771/1615-634x-2000-1-42 
Kitschelt, H. (1994). The transformation of European social democracy. Cambridge: Cambridge University Press.

Kleinen-von Königslöw, K. (2016). Publikumsfragmentierung in der Online-Nachrichtenumgebung. In P. Henn, \& D. Frieß (Hrsg.), Politische Online-Kommunikation. Voraussetzungen und Folgen des strukturellen Wandels der politischen Kommunikation (Digital Communication Research 3, S. 253-278). Berlin. doi:10.17174/dcr.v3.11

Knobloch-Westerwick, S. (2014). Selection, perception, and processing of political messages. In C. Reinemann (Hrsg.), Political communication (Handbooks of communication science, vol. 18, pp. 507-526). Berlin: DeGruyter.

Köcher, R. (2016). Flüchtlingsstrom. Auswirkungen eines gesellschaftlichen Aufregungszyklus auf politisches Interesse und Mediennutzung. Institut für Demoskopie Allensbach. Zugriff am 15.06.2018. Verfügbar unter https://www.ifd-allensbach. de/fileadmin/AWA/AWA_Praesentationen/2016/AWA_2016_Koecher_Fluechtlingskrise_Medien.pdf

Kösters, R., \& Jandura, O. (2018). Politische Kommunikation in heterogenen Lebenswelten. Kommunikationspraxis in politischen Milieus und Bedingungen ihrer Integration. Studies in Communication and Media, 7(3), 129-185. doi:10.5771/2192-4007-2018-2-129

Lee, N.-J., McLeod, D. M., \& Shah, D. V. (2008). Framing policy debates. Issue dualism, journalistic frames, and opinions on controversial policy issues. Communication Research, 35(5), 695-718. doi:10.1177/0093650208321792

López-Escobar, E., Llamas, J. P., \& McCombs, M. (1998). Agenda setting and community consensus. First and second level effects. International Journal of Public Opinion Research, 10(4), 335-348. doi:10.1093/ijpor/10.4.335

Mahrt, M., \& Begenat, M. (2013). Von Lebenswelten und ihren Horizonten. Mediennutzung und Themenwahrnehmung in politischen Milieus. Medien und Kommunikationswissenschaft, 61(1), 21-37. doi:10.5771/1615-634x-2013-1-21
McCombs, M. (2004). Setting the agenda. The mass media and public opinion. Malden, MA: Blackwell.

McCombs, M., \& Shaw, D. L. (1993). The evolution of agenda-setting research. Twenty-five years in the marketplace of ideas. Journal of Communication, 43(2), 58-67. doi:10.1111/j.1460-2466.1993.tb01262.x

Napoli, P. M. (2014). Automated media. An institutional theory perspective on algorithmic media production and consumption. Communication Theory, 24(3), 340-360. doi:10.1111/comt.12039

Neidhardt, F. (1994). Jenseits des Palavers. Funktionen politischer Öffentlichkeit. In W. Wunden (Hrsg.), Öffentlichkeit und Kommunikationskultur (S. 19-30). Hamburg, Stuttgart: Steinkopf.

Neugebauer, G. (2007). Politische Milieus in Deutschland. Eine Studie der Friedrich-Ebert-Stiftung. Bonn: Dietz.

Niedermayer, O. (2009). Gesellschaftliche und parteipolitische Konfliktlinien. In S. Kühnel, O. Niedermayer, \& B. Westle (Hrsg.), Wähler in Deutschland (S. 30-67). Wiesbaden: VS Verlag für Sozialwissenschaften.

Petersen, T., \& Mayer, T. (2017). Ende des Aufruhrs. Wie die Deutschen mit sich selbst Frieden schlossen. Marburg: Tectum.

Prior, M. (2013). Media and political polarization. Annual Review of Political Science, 16, 101-127. doi:10.1146/annurev-polisci-100711-135242

Rippl, S. (2008). Politische Sozialisation. In K. Hurrelmann, M. Grundmann, \& S. Walper (Hrsg.), Handbuch Sozialisationsforschung (S. 443-457). Weinheim, Basel: Beltz.

Roggeband, C., \& Vliegenthart, R. (2007). Divergent framing. The public debate on migration in the Dutch parliament and media, 1995-2004. West European Politics, 30(3), 524-548. doi:10.1080/01402380701276352

Schemer, C., Wirth, W., \& Matthes, J. (2012). Value resonance and value framing effects on voting intentions in direct-democratic campaigns. American Behavioral Scientist, 56(3), 334-352. doi: $10.1177 / 0002764211426329$

Scheufele, B., \& Engelmann, I. (2014). Im Rahmen von Werten. Das Value-Framing der Qualitäts- und Boulevardpresse bei aus- 
gewählten Bundestagswahlen. In F. Marcinkowski (Hrsg.), Framing als politischer Prozess. Beiträge zum Deutungskampf in der politischen Kommunikation (S. 95109). Baden-Baden: Nomos.

Scheufele, B., Kordes, C., Meyer, H., Teutsch, D., Tretter, K., \& Schieb, C. (2012). Garant oder Gefahr. Ein Medienwirkungsexperiment zur Instrumentalität des Value-Framing. Medien und Kommunikationswissenschaft, 60(3), 432-451.

doi: 10.5771/1615-634x-2012-3-432

Schneider, J., \& Eisenegger, M. (fög/Universität Zürich, Hrsg.) (2016). Wie Mediennutzer in die Welt schauen. Die Newsrepertoires der Schweizerinnen und Schweizer und ihre Themenagenden. Studien Qualität der Medien (SQM): 2/2016.

Stark, B. (2013). Fragmentierung Revisited. Eine theoretische und methodische Evaluation im Internetzeitalter. In W. Seufert, \& F. Sattelberger (Hrsg.), Langfristiger Wandel von Medienstrukturen (S. 199-218). Baden-Baden: Nomos.

Stark, B. (2014). Informationsverhalten im 21. Jahrhundert - eine repertoire-orientierte Analyse veränderter Nutzungsmuster. In K. Kleinen-von Königslöw, \& K. Förster (Hrsg.), Medienwandel und Medienkomplementarität aus Rezeptions- und Wirkungsperspektive (S. 37-57). Baden-Baden: Nomos.

Stokes, D. E. (1963). Spatial models of party competition. American Political Science Review, 5(2), 368-377. doi:10.2307/1952828

Stroud, N. J. (2011). Niche news. The politics of news choice. New York: Oxford University Press.

Sunstein, C. R. (2007). Republic.com 2.0. Princeton, Oxford: Princeton University Press.

van Aelst, P., Strömbäck, J., Aalberg, T., Esser, F., Vreese, C. de, Matthes, J. et al. (2017). Political communication in a high-choice media environment. A challenge for democracy? Annals of the International Communication Association, 41(1), 3-27. doi: 10.1080/23808985.2017.1288551

van Hoof, A. M. J., Jacobi, C., Ruigrok, N., \& van Atteveldt, W. (2014). Diverse politics, diverse news coverage? A longitudinal study of diversity in Dutch political news during two decades of election campaigns. $E u$ - ropean Journal of Communication, 29(6), 668-686. doi:10.1177/0267323114545712

Vetter, A. (1997). Political Efficacy. Alte und neue Meßmodelle im Vergleich. Kölner Zeitschrift für Soziologie und Sozialpsychologie, 49, 53-73.

Vlašić, A. (2004). Die Integrationsfunktion der Massenmedien. Begriffsgeschichte, Modelle, Operationalisierung. Wiesbaden: VS Verlag für Sozialwissenschaften.

Vowe, G. (2016). Politische Kommunikation in der Migrationskrise. Der strukturelle Wandel der Kommunikation als Herausforderung für Politik und Wissenschaft. Publizistik, 61(4), 431-440. doi:10.1007/s11616-016-0303-7

Vowe, G. (2017). Wie verändern sich Wahlkämpfe in der Onlinewelt? Sieben Tendenzen des strukturellen Wandels der politischen Kommunikation. Media Perspektiven,12, 607-615.

Weiß, R. (2000). Praktischer Sinn, soziale Identität und Fern-Sehen. Ein Konzept zur Analyse der Einbettung kulturellen Handelns in die Alltagswelt. Medien und Kommunikationswissenschaft, 48(1), 42-62. doi:10.5771/1615-634x-2000-1-42

Weiß, R. (2009). Politisch-kommunikative Milieus. Medien und Kommunikationswissenschaft, 57(1), 1-22. doi:10.5771/1615-634x-2009-1-3

Weiß, R. (2013). Segmentierung politischer Kommunikation in Milieus. In K. Imhof, R. Blum, H. Bonfadelli, \& O. Jarren (Hrsg.), Stratifizierte und segmentierte Öffentlichkeit (S. 205-217). Wiesbaden: Springer VS.

Weiß, R., \& Jandura, O. (2017). Medien und gesellschaftlicher Zusammenhalt. Welche Leistungen öffentlicher Kommunikation braucht eine demokratische Gesellschaft? In O. Jandura, M. Wendelin, M. Adolf, \& J. Wimmer (Hrsg.), Zwischen Integration und Diversifikation. Medien und gesellschaftlicher Zusammenhalt im digitalen Zeitalter (S. 11-31). Wiesbaden: Springer VS.

Weiß, R., Magin, M., Hasebrink, U., Jandura, O., Seethaler, J., \& Stark, B. (2016). Publizistische Qualität im medialen Wandel - eine normativ begründete Standortbestimmung. In P. Werner, L. Rinsdorf, T. Pleil, \& K.-D. Altmeppen (Hrsg.), VerantwortungGerechtigkeit-Öffentlichkeit. Normativität 
in den Medien und in der Kommunikationswissenschaft (S. 27-49). Konstanz: UVK.

Wessler, H. (2002a). Multiple Differenzierung und kommunikative Integration. Symbolische Gemeinschaften und Medien. In K. Imhof, O. Jarren, \& R. Blum (Hrsg.), Integration und Medien (S. 56-76). Wiesbaden: Westdeutscher Verlag.

Wessler, H. (2002b). Reden über Streit. Theoretische Überlegungen zur politischen Konfliktkommunikation in der Mediengesellschaft. In H. Schatz, P. Rössler, \& J.-U. Nieland (Hrsg.), Politische Akteure in der Mediendemokratie. Politiker in den Fesseln der Medien? (S. 189-201). Wiesbaden: Westdeutscher Verlag.

Wolling, J. (2005). Normalzeit vs. Spezialzeit. Besondere Ereignisse als Problem der Stichprobenziehung bei Inhaltsanalysen von Medienangeboten. In V. Gehrau, B. Fretwurst, B. Krause, \& G. Daschmann (Hrsg.), Auswahlverfahren in der Kommu- nikationswissenschaft (S. 138-157). Köln: Halem.

World Values Survey. (2013). WVS Wave 6 (2010-2014). Germany 2013. Zugriff am 15.06.2018. Verfügbar unter http:// www.worldvaluessurvey.org/WVSDocumentationWV6.jsp

Yeo, S. K., Cacciatore, M. A., \& Scheufele, D. A. (2015). News Selectivity and Beyond. Motivated Reasoning in a Changing Media Environment. In O. Jandura, T. Petersen, C. Mothes, \& A.-M. Schielicke (Hrsg.), Publizistik und gesellschaftliche Verantwortung. Festschrift für Wolfgang Donsbach (pp. 83-104). Wiesbaden: Springer VS.

Zaller, J. (2003). A new standard of news quality. Burglar alarms for the monitorial citizen. Political Communication, 20(2), 109-130. doi:10.1080/10584600390211136

\section{Anhang}

Tabelle 5: Themenvalenz pro Milieu

\begin{tabular}{l|c|c|c}
\hline \multirow{2}{*}{ Milieu } & \multicolumn{2}{|c}{ Themenvalenz } \\
\cline { 2 - 3 } & positiv & ambivalent & negativ \\
\hline Sozialdemokratische Mitte & 11.6 & 68.5 & 19.9 \\
Autoritäre Mitte & 4.2 & 43.8 & 51.4 \\
Gemässigt Markt-Autoritäre & 6.2 & 57.9 & 34.5 \\
Staatsskeptische Individualisten & 5.6 & 45.6 & 48.9 \\
Wenig Interessierte & 5.5 & 46.4 & 44.8 \\
Unzufriedene Linke & 6.4 & 55.3 & 37.2 \\
Marktorientiert-Involvierte & 17.0 & 59.5 & 22.2 \\
Sozialautoritäre & 1.7 & 25.9 & 70.7 \\
Engagierte Konservative & 12.5 & 50.0 & 34.4 \\
Prekär-Distanzierte & 0.0 & 46.5 & 44.2 \\
Konkurrenzorientierte Rechte & 2.5 & 28.7 & 68.0 \\
Kritisch-Engagierte & 17.0 & 59.1 & 22.7 \\
\hline
\end{tabular}

Anmerkung: Prozentuelle Anteile; Antworten auf die Frage «Können Sie angeben, ob Sie mit dem Thema ¿Zuwanderung von Flüchtlingen» positive oder negative Gefühle verbinden?»; "weiss nicht» bzw. keine Angabe hier nicht dargestellt. 
Tabelle 6: Ergebnisse Hauptkomponentenanalyse

\begin{tabular}{|c|c|c|c|c|c|c|c|}
\hline $\begin{array}{l}\text { Variable } \\
\text { (prozentualer Anteil erklärter } \\
\text { Varianz) }\end{array}$ & $\begin{array}{l}\text { Faktor } 1 \\
(21.11)\end{array}$ & $\begin{array}{l}\text { Faktor } 2 \\
\text { (14.58) }\end{array}$ & $\begin{array}{l}\text { Faktor } 3 \\
(10.90)\end{array}$ & $\begin{array}{l}\text { Faktor } 4 \\
(10.31)\end{array}$ & $\begin{array}{l}\text { Faktor } 5 \\
(9.24)\end{array}$ & $\begin{array}{l}\text { Faktor } 6 \\
\text { (7.41) }\end{array}$ & $\begin{array}{c}\text { Faktor } 7 \\
(5.71)\end{array}$ \\
\hline Positive Betrachtung & 0.8219 & 0.2148 & 0.0091 & -0.3865 & -0.2469 & 0.0114 & -0.0259 \\
\hline Neutrale Betrachtung & -0.2117 & -0.9634 & 0.0504 & -0.1907 & 0.0084 & -0.1281 & 0.0138 \\
\hline Begrenzung der Zuwanderung & -0.2047 & 0.6999 & 0.0086 & -0.0611 & 0.1666 & 0.1847 & -0.3407 \\
\hline Negative Betrachtung & -0.3334 & 0.6509 & -0.0212 & 0.1156 & -0.0635 & -0.0686 & 0.2528 \\
\hline Multikulturalismus & 0.8949 & 0.1035 & 0.0841 & 0.0519 & -0.1821 & -0.1283 & 0.0302 \\
\hline Empathie für Flüchtlinge & 0.7775 & 0.1634 & 0.0336 & 0.2165 & -0.0630 & -0.1033 & 0.0225 \\
\hline $\begin{array}{l}\text { Nationalismus, Autoritarismus } \\
\text { (schwache Ausprägung) }\end{array}$ & 0.0901 & 0.0324 & 0.0886 & -0.1147 & 0.2146 & 0.0615 & 0.5576 \\
\hline $\begin{array}{l}\text { Nationalismus, Autoritarismus } \\
\text { (starke Ausprägung) }\end{array}$ & -0.0637 & 0.3061 & 0.1405 & 0.2204 & -0.4425 & 0.0051 & 0.2590 \\
\hline $\begin{array}{l}\text { Autoritarismus: Sicherheit } \\
\text { und Kriminalität }\end{array}$ & -0.1178 & -0.0350 & 0.0552 & 0.1591 & 0.2300 & -0.0281 & 0.6921 \\
\hline Autoritarismus: Terrorismus & -0.0090 & 0.4766 & -0.1745 & 0.6746 & 0.0693 & -0.0255 & 0.0040 \\
\hline $\begin{array}{l}\text { Positive Betrachtung aus } \\
\text { sozioökonomischer Perspektive }\end{array}$ & 0.0818 & -0.1178 & 0.0397 & 0.1312 & 0.8598 & 0.1171 & -0.0919 \\
\hline $\begin{array}{l}\text { Neutrale bis tendenziell } \\
\text { negative Betrachtung aus } \\
\text { sozioökonomischer Perspektive }\end{array}$ & -0.1816 & -0.1198 & 0.2428 & 0.1877 & 0.1395 & -0.6624 & 0.3679 \\
\hline $\begin{array}{l}\text { Negative Betrachtung aus } \\
\text { sozioökonomischer Perspektive }\end{array}$ & -0.5878 & 0.1146 & 0.1878 & 0.2626 & 0.1920 & -0.0944 & -0.0716 \\
\hline $\begin{array}{l}\text { Elitenkritik mit Bezug auf Konflikt- } \\
\text { dimensionen }\end{array}$ & 0.1289 & 0.0084 & 0.9357 & -0.0872 & -0.0174 & -0.0481 & 0.1267 \\
\hline $\begin{array}{l}\text { Elitenkritik ohne Bezug auf } \\
\text { Konfliktdimensionen }\end{array}$ & -0.0027 & 0.0944 & -0.9226 & 0.1441 & -0.3109 & -0.2582 & -0.0314 \\
\hline Kritik an einzelnen Politikern & -0.0280 & 0.2558 & 0.0907 & -0.7885 & -0.2304 & -0.1553 & 0.1031 \\
\hline Medienkritik & & -0.2406 & -0.0682 & 0.0352 & 0.1537 & 0.8106 & 0.0108 \\
\hline $\begin{array}{l}\text { Erstarken Rechtspopulismus } \\
\text { und Fremdenfeindlichkeit }\end{array}$ & 0.5433 & -0.3036 & 0.2218 & 0.4469 & -0.2469 & 0.0227 & -0.1852 \\
\hline $\begin{array}{l}\text { Herausforderung für gesellschaft- } \\
\text { lichen Zusammenhalt }\end{array}$ & 0.0988 & -0.2043 & -0.2109 & -0.0226 & -0.6084 & 0.2384 & 0.1757 \\
\hline Regulierung & 0.2152 & -0.0795 & -0.2453 & -0.0200 & 0.1666 & 0.2332 & 0.3348 \\
\hline
\end{tabular}

Anmerkung: In der Tabelle sind Faktorladungen grau hinterlegt, die größer oder kleiner als 0,3 betragen. Erläuterung: Tabelleneinträge sind Faktorladungen einer Strukturmatrix der durchgeführten Hauptkomponentenanalyse mit schiefwinkliger Rotation. Anzahl der Faktoren bestimmt durch KMO-Kriterium mit einem Eigenwert der Faktoren höher als 1. 
